\title{
"Manga-fying" la Commedia: dialogismo testuale e visivo in Dante Shinkyoku di Go Nagai
}

\author{
Raul Ciannella \\ Universitat Autònoma de Barcelona \\ raul.ciannella@e-campus.uab.cat \\ Maria Antònia Martí Escayol \\ Universitat Autònoma de Barcelona \\ mariaantonia.marti@uab.cat
}

\section{Riassunto}

L'articolo analizza Dante Shinkyoku, ダンテ神曲 (La Divina Commedia di Dante, 1994) un adattamento manga del poema dantesco realizzato da Go Nagai. Attraverso lo studio degli elementi strutturali, impiegati da Nagai per sostenere l'adattamento, e dei meccanismi di significazione e rappresentazione del linguaggio proprio del manga, sveleremo la molteplicità di livelli (storici, estetici, narrativi, discorsivi, ecc.) attraverso cui l'adattamento, in quanto processo interpretativo e creativo, si configura come relazione dialogica con l'ipotesto e al contempo come prodotto in sé.

Parole chiavi: adattamento; dialogismo intertestuale; Dante; Dante Shinkyoku; Divina Commedia; Gustave Doré; Go Nagai; Linguaggio Visivo Giapponese; Manga.

\begin{abstract}
In this article we will examine Dante Shinkyoku, ダンテ神曲 (The Divine Comedy of Dante, 1994), Go Nagai's manga adaptation of the Italian poem. Through the study of both the structural elements upon which the adaptation is sustained and manga's language inherent signifying and representational mechanisms, we will unveil the multiplicity of levels (historical, aesthetic, narrative, discursive, etc.) through which the adaptation is configured, establishing a dialogical relation with the hypotext and, at the same time, asserting its own uniqueness.
\end{abstract}

Keywords: adaptation; Dialogic intertextuality; Dante; Dante Shinkyoku; Divine Comedy; Gustave Doré; Go Nagai; Japanese Visual Language; Manga. 
J el 1994 Go Nagai pubblica per la casa editrice Kodansha di Tokio La 1 Divina Commedia di Dante (Dante Shinkyoku, ダンテ神曲) ${ }^{\mathrm{I}}$ la prima versione manga del poema dantesco.

Go Nagai (pseudonimo di Kiyoshi Nagai, 永井潔, 1945, Wajima, Ishikawa) è uno dei più influenti mangaka giapponesi. A lui dobbiamo le popolarissime serie di Super Robot, i mecha giganti e ipertecnologici controllati da piloti umani al loro interno, come Mazinger Z(マジンガーZ, Majingā Zetto, 1972), Il grande Mazinger (グレートマジンガー, Gurēto Majingā, 1974), UFO Robot Goldrake (UFOロボグレンダイザー, UFO Robo Gurendaizā, I975) e Jeeg robot d'acciaio (鋼鉄ジーグ Kötetsu Jìgu, 1975). Verso la fine degli anni settanta questi ed altri personaggi invasero letteralmente l'Europa e in particolare l'Italia, che tra il 1978 e il 1983 aveva già importato 183 serie di anime (Malone 20I0: 317). Da allora, sia i manga che gli anime realizzati da Nagai continuano a riscuotere un enorme successo in Italia, dove è celebrato come uno dei maestri indiscussi di questo medium.

Ma il legame tra Nagai e l'Italia ha radici più profonde che vanno ricercate nella sua relazione con la Commedia, di cui lesse, ancora fanciullo, una versione per ragazzi illustrata da Gustave Doré. In numerose dichiarazioni e interviste, Nagai ammette l'impatto che quella lettura e quelle illustrazioni ebbero nella costruzione del suo immaginario e nella ricerca tematica e stilistica che costituiranno la base della sua poetica, combinandosi con altri elementi propri della cultura giapponese, come le opere del maestro Osamu Tezuka e il cinema fantascientifico giapponese degli anni cinquanta.

L'idea di realizzare una versione manga della Commedia risale ai primi anni settanta quando Nagai, reduce dal grande successo del controverso La scuola senza pudore (ハレンチ学園, Harenchi Gakuen, 1968) riceve carta bianca dalla casa editrice Kodansha per realizzare una storia che avrebbe inaugurato la nuova rivista settimanale Bokura Magazine: "fu in quel momento che la Divina Commedia mi risalì alla memoria, tuttavia ritenni che farne semplicemente una versione a fumetti non sarebbe stato interessante e così decisi di aggiungervi degli elementi di fantascienza e di assumere non il punto di vista di Dante bensì dei demoni." (Nagai 1998a: 3)

Nasceva così Mao Dante (魔王ダンテ, Maō Dante, 197I) un manga che dichiarava sin dal titolo il suo vincolo con il poeta, ma soprattutto con le illustrazioni di Doré (di cui riprendeva numerosi motivi visivi come il vortice, la rappresentazione dei demoni, il demone intrappolato tra i ghiacci). Si

I. Le pagine citate in questo lavoro si riferiscono tutte all'edizione giapponese in due volumi uscita nel 1998 (Nagai 1998b). Abbiamo utilizzato inoltre, per i riferimenti testuali, l'edizione italiana in tre volumi pubblicata da Edizioni BD/J-Pop nel 2014 su licenza esclusiva della Dynamic Planning (Nagai 20I4) la quale però non possiede numeri di pagina. Ci atterremo quindi, anche in questo caso, alle tavole equivalenti della sopracitata edizione giapponese. Un ringraziamento a Jordi Besa per le traduzioni. 
trattò di un'appropriazione che ribaltava i valori espressi dalla Commedia, dal momento che giocava sull'ambiguità tra il bene e il male attraverso un rovesciamento onomastico e prospettico. Infatti, in Mao Dante Dio è un'essenza cosmica maligna e distruttiva, seminatore del male e causa dell'annientamento delle città pacifiche e lussureggianti di Sodoma e Gomorra. Al contrario, il demone Dante, ibridato con l'adolescente Ryo Utsugi, e la sua alleata, Medusa, sono descritti come eroi positivi. Mao Dante rimarrà incompiuto per via dell'interruzione prematura della rivista, ma costituirà il nucleo sul quale si fonderà uno dei personaggi più popolari di Nagai, Devilman (デビルマン, Debiruman, 1972).

Attraverso Mao Dante e Devilman, Nagai costruisce una vera e propria mitologia fatta di richiami intertestuali, echi onomastici e motivi visivi che si rifanno sia alla Commedia che alla tradizione greco-romana e che il mangaka proseguirà anche nelle sue opere successive di taglio più fantascientifico (Scilabra 20I5: IOI-IO3). Dovranno invece passare più di vent'anni dalla pubblicazione di Mao Dante perché Go Nagai possa finalmente realizzare l'idea primigenia di un adattamento manga del poema dantesco.

\section{La Divina Commedia di Dante}

Fu il letterato Ōgai Mori (1862-1922) a coniare, verso la fine dell'Ottocento, l'espressione Shinkyoku (Canto Divino) per designare il poema dantesco, espressione con cui tuttora si conoscono le traduzioni della Commedia al giapponese. Adottando lo stesso titolo, Nagai s'inserisce quindi nella stessa tradizione letteraria. Ma se da una parte il titolo denota la relazione diretta con il testo fonte, dall'altra risulta chiaro fin dalla prima pagina che ci troviamo di fronte a una delle "infinite avventure dell'interpretazione" (Eco 20I2: I3.8).

Dante Shinkyoku non è (o perlomeno non è solo) il risultato di una volontà di recuperare un testo della letteratura canonica occidentale e trasmutarlo in un altro medium per renderlo accessibile ed appetibile ad un pubblico culturalmente distante. Si tratta piuttosto di un atto "creativo e interpretativo di appropriazione" (Hutcheon 2006: 8) che rivela la molteplicità di livelli (storici, estetici, narrativi, discorsivi, ecc.) attraverso cui vari sistemi culturali, in quanto entità dinamiche, entrano in dialogo, si sovrappongono e si riconfigurano. L'adattamento quindi, nella sua doppia accezione di "processo e prodotto" (ibid.: 9), da una parte si configura come una serie di operazioni di transcodifica da un sistema semiotico ad un altro, dove il mangaka funge da "traduttore visibile" (Şerban 20I4: 23); dall'altra mostra come questo processo non può avvenire in un vuoto, né tantomeno in maniera meccanica o innocente, bensì in un contesto culturale, sociale e storico determinato, seguendo il gusto, le motivazioni, gli interessi e le finalità di chi adatta e innescando una 
complessa "grammatica della trasformazione" che comprende operazioni di selezione, amplificazione, concretizzazione, critica, estrapolazione, analogizzazione, popolarizzazione e transculturazione (Stam 2000: 68).

La nostra analisi verterà quindi su due aspetti principali che svelano questa relazione dialogica e al contempo mostrano Dante Shinkyoku come prodotto in sé, "oggetto palinsestico di se stesso" (Hutcheon 2006: 9): in primo luogo, esamineremo gli elementi strutturali impiegati da Nagai per sostenere l'adattamento (riproduzione delle litografie di Gustavo Doré su cui "appoggiano" le sequenze narrative, eliminazione del Dante auctor come atto di appropriazione); in secondo luogo osserveremo come i procedimenti formali propri del manga attivino i meccanismi di significazione in relazione alla trasmutazione dei versi danteschi.

\section{INTRODUZIONE AGLI STUDI SUL MANGA}

Nell'ambito della teoria del manga, già a partire dagli anni novanta, si è andata delineando una linea di studi denominata manga hyōgenron, traducibile come teoria dell'espressione o della rappresentazione del manga, proposta da autori come Natsume Fusanosuke e Takekuma Kentarō (Fusanosuke 2003; Fusanosuke 2013), il cui scopo è l'elaborazione di strumenti metodologici per un'analisi formale del manga, considerato come un medium espressivo-rappresentativo autonomo e distintivo. Partendo da Fusanosuke, ma integrando anche gli approcci più recenti che sfruttano la teoria dei sistemi, il critico Itō Gō ha sviluppato la manga hyōgen shisutemu-ron, (teoria del sistema di rappresentazione del manga; Gō 2005) che incorpora anche l'estetica della ricezione (Gō 20II: 75). Similmente, altre linee di ricerca esaminano aspetti attinenti all'esperienza visiva, l'alfabetizzazione, i processi di apprendimento e le pratiche rappresentazionali (Nakazawa 2002; Ingulsrud-Allen 20I0), i vincoli tra ricezione e funzione autoriale, un aspetto denotato specialmente nei fenomeni otaku e moe (Kinsella 2000: I28s; Galbraith 20I4) o le specificità dei dialetti propri di ogni distinta tipologia di manga (definita per frangia d'età e genere del pubblico lettore) così come delle traduzioni o del fenomeno scanlator (Inoue 2009; Fujimura 2012).

Orientativamente, gli studi che conformano il manga hyögenron si focalizzano sugli elementi morfologici, sintattici e semantici che conformano il linguaggio proprio del manga ed i processi cognitivi ad essa associati.

Parallelamente, il linguista e neurologo statunitense Neil Cohn ha sviluppato una metodologia basata sull'analisi del Linguaggio Visivo Giapponese, ovvero di quegli elementi grafici e verbali, da lui definiti morfemi visivi, contenuti in ogni vignetta; e della Grammatica della Narrativa Visiva, ovvero delle strutture sintattiche in cui la narrazione si organizza. Cohn parte dagli 
studi semiotici sul fumetto elaborati da Scott McCloud e li integra ed estende con elementi ispirati alla grammatica generativa di Chomsky, alla psicologia cognitiva e alla neuroscienza (McCloud I993; Cohn 20I0).

Cohn classifica i morfemi visivi in base alla strategia combinatoria che adottano, suddividendoli in: affissi, morfemi che si aggiungono ad altri morfemi definendo il loro stato (ad esempio una stella sulla testa di un personaggio può indicare sorpresa); morfemi di reduplicazione (per esempio, disegnare due teste di uno stesso personaggio mostra un cambio di umore); morfemi di soppiantamento, che sostituiscono un altro morfema (per esempio, due cuori al posto delle pupille di un personaggio manifestano un innamoramento repentino); e gli sfondi, che attraverso l'uso di colori, forme e linee possono trasmettere emozioni e stati d'animo o proiettare all'esterno la soggettività del personaggio.

Per quanto riguarda la Grammatica della Narrativa Visiva, Cohn prende spunto dalle conclusioni di Scott McCloud sulla sequenzialità, ovvero il modo in cui le vignette si relazionano tra loro facendo procedere la narrazione. McCloud si sofferma sulle relazioni tra una vignetta e la successiva proponendo sei tipi di transizioni: "da momento a momento", "da azione ad azione", "da soggetto a soggetto", "da scena a scena", "da aspetto ad aspetto" (molto frequente nel manga) e "non-sequitur" (McCloud 1993: 70-74). In alternativa a quest'analisi lineare, Cohn propone di prendere in esame strutture più ampie che definisce "archi" e che includono diverse categorie narrative paragonabili a quelle sintattiche che compongono una frase. Le categorie principali che individua sono: l'Establisher, che introduce passivamente le entità partecipanti nell'arco; l'Initial, che marca il punto d'inizio dell'azione o della tensione narrativa; il Prolongation, un'estensione spaziale e/o temporale che si colloca tra l'Initial e il Peak; il Peak, dove si concentra il punto massimo della tensione narrativa; e il Release, che indica il momento in cui la tensione dell'arco decade. Inoltre, ognuna di queste categorie può essere accompagnata da Refiners, modificatori che focalizzano l'attenzione su dettagli specifici dell'azione per evidenziarne l'importanza (Cohn 20I0: 196).

Altri studi, che riguardano sia il manga sia il fumetto in generale, sottolineano inoltre l'importanza narrativa ed espressiva del framing, ovvero della forma e della disposizione spaziale delle vignette sulle tavole. Oltre ai già citati Fusanosuke, Cohn e McCloud va sicuramente menzionato il lavoro pioneristico di Will Eisner (1985: 38-6I).

Per la nostra analisi formale adotteremo soprattutto la terminologia e le metodologie proposte da Cohn e McCloud, concentrandoci sui tre elementi sopramenzionati: i morfemi visivi, la sequenzialità e il framing. 


\section{Elementi strutturali in Dante SHINKYokU}

"Ero ragazzo quando i miei fratelli portarono a casa un'edizione della Divina Commedia illustrata da Gustave Doré. Desiderai immediatamente poter disegnare come faceva lui" (Prisco 2007). Come risulta evidente da questa dichiarazione il fascino che la Commedia esercita su Nagai deriva innanzitutto dalla suggestione visiva che le illustrazioni di Doré gli trasmettono. "Fu soprattutto l'immagine di Lucifero tricipite incastrato nel ghiaccio a colpirmi tanto da rimanermi per sempre incisa nella memoria" (Nagai 1998: 3). L'incisore francese diviene così il riferimento diretto e al tempo stesso l'intermediario necessario tra Nagai e il poema, determinando l'impostazione stilistica e iconografica del manga. Dalla lettura della Commedia invece, il mangaka trarrà materia tematica con la quale indirizzare la linea narrativa dell'adattamento, improntata ad un'analisi del lato oscuro dell'essere umano e allo sviluppo della relazione tra Dante e Beatrice.

Esiste abbondante bibliografia sulla crescente popolarità di Dante in Europa a partire dal XIX secolo e sul ruolo che Doré ebbe sia nell'accrescere questa popolarità nel mondo, sia nella composizione di un'iconografia del poema dantesco nelle arti visive e nell'immaginario popolare. ${ }^{2}$ Ed è proprio su questo ultimo aspetto che le illustrazioni di Doré mostrano la loro straordinaria forza d'impatto, come sottolineava il rinomato e sagace commento di Daniele Mattalia: "chi approdava ai versi di Dante dopo essersi imbevuto di Doré, era quasi come se leggesse ampie didascalie. La selva selvaggia aveva quelle radici di querce scoperte, quei rovi; Caronte aveva quella barba; Minosse aveva quella coda; Farinata aveva quel lastrone dietro la schiena, aveva quel costato, Farinata era sulla sinistra, Virgilio e Dante stavano sulla destra" (Mattalia 1973: LXVIII).

Ed è quel mondo illustrato da Doré a costituire la base dell'ambientazione del manga. Basta aprire Dante Shinkyoku in un qualunque punto per rendersi conto della funzione di Doré nella realizzazione del manga sia a livello strutturale che stilistico. Le illustrazioni dell'incisore francese sono riprodotte quasi totalmente ${ }^{3}$ e disposte lungo la linea narrativa come pietre miliari, costituendo la vera e propria ossatura dell'opera. In molti casi, la riproduzione calca esattamente l'immagine fonte senza cambi sostanziali, tranne lievi ritagli o aggiustamenti dovuti alla composizione. In altri casi, come vedremo più avanti, le modifiche sono più incisive, per rispettare la grammatica del manga, aggiungere dinamismo alla narrazione o restituire movimento e azione pur presenti nei versi danteschi, però poco rappresentati nelle litografie.

2. Punto di riferimento essenziale in questo senso è costituito dalle ricerche di Aida Audeh, tra cui consigliamo: Audeh 2009.

3. Delle settantacinque litografie che Doré realizzò per l'Inferno, Nagai ne riproduce sessantatré; quaranta su quarantadue per il Purgatorio e tredici su diciotto per il Paradiso. 
Il disegno delle tavole è realizzato con la tecnica del tratteggio per imitare l'effetto della litografia, una scelta stilistica che, come commenta lo stesso Nagai, non fu facile, vista la quantità di linee necessarie per completare una tavola, che incrementavano notevolmente le ore di lavoro (Nagai 1998: 36I). La narrazione invece procede attraverso una serie di accorgimenti tecnici e innesti intertestuali che sembrano riempire lo spazio diegetico tra un'illustrazione di Doré e l'altra. È in questo spazio che avvertiamo con più forza il poema dantesco, che giunge sì come un effetto secondario, ma rivela anche un'attenta lettura da parte di Nagai, che in molti casi riesce nell'ardua impresa di "mappare" i versi della Commedia sul medium grafico del manga.

Anche Nagai, come Doré, è attratto dall' Inferno molto più che dalle altre due cantiche. Le tavole dedicate all'Inferno sono infatti ben 506 rispetto alle I89 del Purgatorio e le sole 68 del Paradiso, dimostrando di adattarsi anche proporzionalmente al lavoro di Doré piuttosto che all'equilibrio formale e simbolico delle tre cantiche.

Affidare un ruolo predominante all'Inferno sembra una scelta scontata e popolare, ma anche affine alla predilezione di Nagai per i mondi sotterranei, le divinità e gli esseri ctoni e la spettacolarizzazione della violenza e della sensualità. Le suggestioni plastiche, sensoriali e drammatiche contenute nelle incisioni di Doré vengono spinte al grado massimo di estrapolazione per enfatizzare, in ogni scena, gli elementi truculenti, patetici e sensuali che il mangaka non perde occasione di esibire.

Ma oltre a costituire un'esperienza sensoriale maggiore e narrativamente più seducente rispetto alle altre due cantiche, crediamo che la scelta di Nagai sia anche dettata da un realismo che l'Inferno è in grado di esprimere in maniera più universalmente riconoscibile. Eric Auerbach ha dimostrato, attraverso il suo concetto di "realismo figurale", come tutta la Commedia sia permeata da un realismo intenso, in quanto concentrato nella realizzazione piuttosto che nella figura, e ciò proprio a causa della condizione di atemporalità e immutabilità (changeless existence, Auerbach 2003: 19I) di cui le anime della Commedia sono consapevoli. Ma Auerbach ammette anche che il rimestio emotivo causato da questa consapevolezza è più intenso nell' Inferno (Auerbach 2003: 193) perché l'anima dannata non può guardare in alto, verso Dio, ma può solo rivolgersi alla vita terrena sotto forma di ricordi (passato) o di premonizioni (futuro). Nel Purgatorio invece, e soprattutto nel Paradiso edificato seguendo la cosmologia aristotelico-tolemaica ed organizzato rispettando il De coelesti hierarchia dello Pseudo Dionigi Aeropagita, è più marcatamente evidente la dottrina cristiana neoplatonica, una dottrina necessariamente lontana nel tempo e nello spazio dalla cultura giapponese ${ }^{4}$ e di cui Nagai, in più punti del

4. “Stavo lavorando alle mie prime opere e avere avuto accesso all'intera mitologia del Cattolicesimo con un'opera così visionaria [la Bibbia di John Huston n.d.a] è stata una 
manga, si fa lettore critico, rifiutando apertamente certe posizioni del poema (Nagai 1998: I, 363)

È l'Inferno quindi, la parte dell'aldilà che costituisce in maniera più intensa "il palcoscenico per gli esseri umani e le loro passioni" (Auerbach 2003: 20I) attraverso un realismo la cui rilevanza è sottolineata anche da alcuni commentatori giapponesi di Dante, come lo scrittore e critico Masamune Hakuchō che in Dante ni tsuite (Su Dante) sosteneva l'incompatibilità del poeta con il Giappone, ma al tempo stesso ne ammirava "l'accuratezza delle scene ed il realismo con cui è rappresentato l'animo umano nelle sue pulsioni più oscure, similmente a un romanzo moderno" (Capponcelli 20I4: 77).

Nagai da una parte semplifica questo realismo e dall'altra lo rende ancora più manifesto attraverso la fusione del Dante biografico col Dante personaggio e l'invenzione di una causalità "logica" per lo sviluppo della narrazione "fantastica”. Infatti, la complessa architettura cosmologica e l'impianto simbolicoallegorico della Commedia svaniscono nella trasmutazione in Dante Shinkyoku che si apre con una "veduta" di Firenze e, dopo un breve ma significativo incipit, che commenteremo più avanti, collega l'episodio biografico dell'esilio da Firenze con la fuga attraverso un bosco (selva oscura) situato fuori dalla città dove il poeta si smarrisce. Una volta nella selva oscura, sia il personaggio che il lettore si immergono effettivamente in un luogo altro, liminare, come da tradizione mitico-fiabesca, ma non in un paesaggio allegorico.

L'episodio biografico viene impiegato quindi come un espediente narrativo a supporto della causalità realista, una tecnica che sarà utilizzata regolarmente. Così, le invettive e le questioni riguardanti il contesto socio-politico contemporaneo a Dante, quando non vengono eliminate, come nel caso della profezia di Ciacco, svolgono la funzione di mantenere la continuità biografico-realista del personaggio piuttosto che rispecchiare la posizione critica del poeta. I dilemmi etico-morali non svaniscono, ma sono attualizzati e affidati al Dante personaggio in maniera inedita rispetto al poema, riverberando problematiche più vicine all'autore giapponese. Anche il Dante di Nagai, come il Dante agens della Commedia è curioso e prono a subissare Virgilio di domande, ma è anche più propenso al dubbio, al tentennamento e al questionamento sia dell'operato umano che divino. Nel "Terzo cerchio" ad esempio, Dante chiede a Virgilio se il peccato di gola sia tanto grave da meritare l'atroce punizione di essere squartati e divorati eternamente da Cerbero. ${ }^{5}$ Virgilio replica in maniera decisa, spostando la dimensione del peccato da un piano individuale a uno so-

benedizione. Dovete pensare che tanti anni fa il Cattolicesimo non era poi così noto in Giappone, per noi era qualcosa di assolutamente esotico". (Danesi 2016)

5. Rispetto alla Commedia si esalta la crudeltà e la violenza del can Cerbero, un mostro gigante che divora le anime per poi espellerle sotto forma di liquame, il quale poi torna a prendere forma umana dando un nuovo inizio alla pena. 
ciale: "I golosi sono peccatori! Non dimenticare che all'ombra di chi s'ingozza vi sono coloro che muoiono di fame!" (Vol. I, p. I33).

In un altro esempio, nella palude dello Stige dove dimorano "l'anime di color cui vinse l'ira" (If. VII v. II7) Nagai traspone egregiamente le lamentele dell'insoddisfazione dei dannati, racchiudendole in bolle che "fanno pullular quest'acqua al summo" (If. VII v. I20). Osservando le bolle, Dante domanda a Virgilio se quell'insoddisfazione non sia piuttosto un'emozione necessaria per affrontare il mondo. Virgilio risponde che l'insoddisfazione non è un peccato in sé, ma lo può diventare a seconda di come la si affronta (Vol. I, p. I79). Un certo grado di critica sociale è sempre presente nelle opere di Nagai, anche in quelle più diffuse e commerciali, situandosi in linea con le proposte di rinnovazione del manga a partire dagli anni sessanta, come il komaga e il gekika, che consideravano imprescindibile il coinvolgimento del medium con il sociale (Rosenbaum 2012: 73).

I dubbi, la paura e la sofferenza di Dante sono condivisi dal lettore grazie a un linguaggio visivo capace di suscitare un senso di immediatezza che rende il fruitore partecipe attivo di un'esperienza diretta, collocata nel presente. Così, nonostante manchi una certa coerenza nella voce narrante, che cambia spesso di persona e di tempi verbali, la grammatica visiva specifica del manga, in maniera per certi versi analoga a quella cinematografica, colloca la vicenda sempre nel "qui e ora", facendo di Dante un personaggio ancora ignaro del percorso che lo aspetta. Nagai impiega tutto l'arsenale che il linguaggio del manga gli mette a disposizione per aumentare l'empatia e l'identificazione col protagonista: l'impiego di primissimi piani e dettagli del volto carichi di pathos; l'uso del masking effect che si basa sul contrasto tra un soggetto rappresentato in forma semplificata e minimalista rispetto a un'ambientazione realista o a personaggi disegnati con maggiore dettaglio, un espediente che aumenta l'empatia del lettore (McCloud 1994: 42-44); l'uso di sfondi "espressionisti" o linee di movimento soggettive che concentrano l'attenzione sul protagonista e "mostrano" la sua attività emotiva o psicologica; l'adozione di un punto di vista soggettivo o di linee cinetiche applicate allo sfondo anziché al personaggio, per incrementare nel lettore la sensazione di partecipare all'azione.

Tutto ciò si associa alla caratterizzazione grafica di Dante, che serve per delineare le emozioni predominanti del personaggio: l'incertezza, la sorpresa, il dubbio. Nagai riprende l'iconografia dantesca classica, che parte da Boccaccio e Giotto e arriva fino a Doré, e su questa realizza ciò che alcuni autori hanno definito mangafication o manga-fying (Lanier 20IO: II2; Şerban 20I3; Şerban 20I4; Jones 20I5; Miyake 2008). Attraverso l'uso di morfemi visivi il ritratto idealizzato di Dante si converte in un personaggio vivo, emotivo e dinamico. Sia nell' Inferno che nel Purgatorio, Dante è accompagnato da gocce di sudore, 
lacrime e ombre sul volto o sotto gli occhi; nel Paradiso invece il sudore sparisce e le lacrime accompagnano un sorriso di felicità (Tav. I).

L'opera di Nagai quindi, in quanto prodotto di un medium che combina parole e immagini, opera un "processo dialogico continuo" (Stam 2000: 64) sia con l'ipotesto verbale, la Commedia, che con quello grafico, le illustrazioni di Doré (a loro volta adattamento della Commedia), mostrando la sua natura palinsestica multilivello. Ma il dialogismo intertestuale si spinge molto oltre, intessendo nelle maglie compositive del manga una serie di citazioni più $o$ meno palesi e dichiarate, sia di natura artistico-pittorica che verbale.

Nel capitolo intitolato "Beatrice", corrispondente al Canto II dell'Inferno, Virgilio rivela a Dante che è stata Beatrice a chiedergli di salvarlo e accompagnarlo nel suo viaggio ultraterreno. A questo punto, un'analessi che attinge direttamente la sua composizione dalla Vita Nuova, espone l'origine della relazione romantica tra il poeta e Beatrice. Attraverso la "decostruzione" e ricomposizione in diverse vignette del dipinto di Henry Holiday Dante and Beatrice (I883), Nagai re-immagina il loro incontro amoroso, capovolgendo il senso stesso del quadro, che si ispira invece al capitolo X della Vita Nuova, nel quale Beatrice nega il saluto a Dante (Fig. I). L'analessi si conclude con una vignetta che riproduce direttamente il frontespizio dell'editio princeps ${ }^{6}$ della Vita Nuova, affiancata da una breve didascalia che spiega l'origine dell'opera. Questo episodio serve, da una parte per continuare con la strategia di fusione tra Dante personaggio e Dante biografico già rilevata, e dall'altra per innescare una delle principali linee tematiche del manga, ovvero il ricongiungimento tra Dante e Beatrice. Per Nagai infatti Beatrice non è né teologia, né allegoria, ma donna in carne e ossa conosciuta da Dante e ritrovata post mortem nel Paradiso.

Differente è invece l'uso di un altro dipinto nella sequenza che narra l'incontro tra Dante e Farinata degli Uberti. Questa volta, in una vignetta che occupa i quattro quinti della tavola, Nagai riproduce Farinata degli Uberti alla battaglia di Montaperti (1842) di Giuseppe Sabatelli. Il rifacimento è accompagnato da una didascalia esplicativa del personaggio con una chiara funzione educativo-didattica su cui ci soffermeremo più avanti. Identica funzione educativa, anche se meno esplicita, assolve un'illustrazione introdotta nel capitolo "Il settimo cerchio" quando Dante e Virgilio s'imbattono nel centauro Chirone. Virgilio commenta a Dante che Chirone fu il maestro di Achille e la tavola successiva è riempita interamente da un'illustrazione di Achille inquadrato di spalle, in groppa ad un cavallo impennato (o lanciato al galoppo). L'eroe, vestito come un soldato romano, brandisce una spada ed ha una freccia conficcata nel tendine del piede destro. Sebbene non sia possibile accertarne la

6. La vita nuova di Dante Alighieri. Con XV. Canzoni del medesimo. E la vita di esso Dante scritta da Giovanni Boccaccio, Firenze, Bartolomeo Sermartelli, 1576. 
fonte, l'anomala iconografia di questa illustrazione ricorda la scultura "Achille a cavallo" realizzata da Domenico Umberto Diano (I887-1977) per la città di Chieti. ${ }^{7}$

Un ultimo quanto significativo esempio di citazione esplicita si produce nel Cocito quando i due poeti giungono al cospetto di Lucifero incastrato tra i ghiacci. Nagai dapprima riproduce esattamente l'illustrazione di Doré che tanto lo colpì da ragazzo, in una vignetta a doppia tavola e senza modifiche. Successivamente, attraverso la voce di Virgilio, riassume la storia di Lucifero, che da bellissimo angelo si convertì ne "lo'mperador de lo doloroso regno" (If. XXXIV v. 28). Le vicende riassunte sono tratte dal Paradiso Perduto, così come le tavole che le accompagnano, in gran parte costituite da rifacimenti delle illustrazioni che lo stesso Doré realizzò per il poema di Milton (Fig. 2).

Questi pochi esempi mostrano la complessa e intricata relazione intertestuale tra la Commedia e Dante Shinkyoku, dove gli interventi di selezione, scarto, rielaborazione e metamorfosi operano su piani multidimensionali, al tempo stesso transtemporali e transculturali. Nagai dialoga con il "mondo" Divina Commedia, un sistema complesso che concatena testi provenienti da società, epoche, culture e luoghi distinti e con il quale il mangaka necessariamente realizza un'operazione di "riduzione della complessità", nel senso che gli attribuisce Anthony Pym, ovvero un'operazione decisionale tra differenti possibilità interpretative e che fornirà la base costitutiva della (ri)creazione (Pym 2004: punto I e 2). In questo senso potremmo adottare ed estendere al manga la definizione di adattamento cinematografico come "negoziazione intertestuale multilivello" proposta da Robert Stam (2000: 67). Un movimento a spirale caratterizza il processo di negoziazione, dove il "mondo Divina Commedia" contribuisce a modellare e definire lo stile, i motivi visivi e le scelte tematiche che ritroviamo disseminati nelle opere di Nagai, poi riconfigurati e ricodificati (ma riconoscibili da un pubblico lettore già alfabetizzato) nella trasposizione intersemiotica del poema, che rientra quindi nello stesso mondo che l'ha generata. Ma il movimento non si ferma qui, giacché Dante Shinkyoku subisce un nuovo processo di transculturazione nelle traduzioni e nell'accesso al mercato europeo e nordamericano. Ogni spira si configura come un nuovo processo e ad ogni spira la relazione polisistemica si intensifica.

7. È possibile che in uno dei suoi numerosi viaggi in Italia dove già dai primi anni ottanta è considerato maestro del manga e dell'anime, Nagai possa aver visto questa scultura. Lui stesso dichiara di trarre ispirazione per i suoi manga dalle sue visite nelle città italiane: "Miti greci e romani sono al centro della mia produzione di manga. Per questo mi emoziona passeggiare per le strade di città come Roma o Napoli, ancora cariche di quelle suggestioni culturali. Al Museo archeologico di Napoli, per esempio, ho visto un guerriero greco in armatura che potrebbe fare da modello per un mio robot..." Cit. 2007. 


\section{ANALISI DELL'ARCO NARRATIVO NELL' INCIPIT}

Per esemplificare quanto esposto finora, analizzeremo l'incipit dell'opera dove sono già evidenti sia l'impianto strutturale che le strategie narrative impiegate da Nagai, una specie di dichiarazione d'intenti che vale per tutta l'opera (Fig. 3). Adotteremo la metodologia di analisi della Grammatica Narrativa Visiva proposta da Neil Cohn (2013).

La sequenza iniziale di Dante Shinkyoku è preceduta da una pagina splash che include il titolo del primo capitolo "La selva oscura di Firenze" ed una immagine panoramica della città. Abbiamo già osservato come l'uso di questa immagine serva da espediente per determinare uno spazio realista, concretizzando geograficamente l'azione. La città è rappresentata attraverso un'icona stereotipica, la cattedrale di Santa Maria del Fiore, la cui cupola di Brunelleschi, costruita nel XV secolo, costituisce un anacronismo necessario per far sì che lo spazio sia riconoscibile dai fruitori del manga. L'arco narrativo vero e proprio inizia con la vignetta successiva, un Establisher che introduce ambiente e personaggi della narrazione: un uomo in mezzo ad una "selva". La vignetta, verticale con inquadratura a piombo su una foresta d'alberi fittissima, senza spazi vuoti, dove a mala pena distinguiamo una figura umana, serve a determinare immediatamente l'atmosfera dell'azione.

Alcune onomatopee indicano il movimento faticoso di quest'uomo minuscolo, perso tra la frondosità e la profondità del bosco. Le due vignette successive attuano da Refiners. La prima è allargata orizzontalmente e immerge chi guarda all'interno del bosco invitandolo a percorrerla. La seconda mostra un dettaglio delle gambe dell'uomo che avanza, un primo avvicinamento al personaggio.

La vignetta successiva è un Initial che mostra un mezzo primo piano dell'uomo di cui però vediamo solo la metà inferiore del viso. Per la prima volta "sentiamo" le sue parole che comunicano incertezza e smarrimento. A seguire, una Prolongation che dilata il tempo e mantiene il mistero sull'uomo, mostrato ora di spalle, con un cappuccio e una corona d'alloro che gli cinge la testa, mentre osserva la nebbiosa selva. La vignetta, statica e muta, occupa la metà inferiore della pagina e fornisce uno spazio mentale per la contemplazione dell'ambiente.

La tavola successiva si apre con il Peak, il punto di tensione più alto, composto da due piccole vignette accostate, di breve durata e transizione rapida che mostrano finalmente il volto dell'uomo, disorientato, mentre si chiede dove sia e perché si trovi in quel luogo. Infine, il Release libera tutta la tensione dell'arco narrativo in un'ultima vignetta di grande respiro e lunga durata che occupa più della metà della pagina sinistra. 
Se nel Prolongation l'uomo era mostrato di spalle, immobile e in silenzio, il Release adotta una posizione spazialmente e concettualmente speculare. L'uomo gira la testa verso il lettore, una doppia nuvoletta permette di leggere sia i suoi pensieri che le sue parole: una rielaborazione parafrasata del primo verso della Commedia. Ora riconosciamo in questa vignetta un adattamento della prima illustrazione dell'Inferno realizzata da Doré, lievemente ravvicinata e con la posizione della testa del poeta invertita. È la fine dell'incipit che dà il via al manga, dove convergono Dante poeta, Doré e naturalmente Nagai, concretando una sintesi che riassume la metodologia di adattamento di tutto il manga, il primo pezzo della struttura che sosterrà l'intera opera. Da notare che l'identità del personaggio verrà espressamente rivelata solo nella tavola successiva, con un primo piano del poeta stesso che si presenta "Io sono Dante... Dante Alighieri” e dà inizio al racconto del suo esilio.

\section{DANTE AUCTOR VS NAgAI AUCTOR}

L'indizio forse più importante della consapevolezza di un'appropriazione del testo da parte di Nagai è l'eliminazione del Dante auctor, ${ }^{8}$ sostituito da un'altra voce impersonale che interrompe la diegesi con commenti, delucidazioni e digressioni, una sorta di Nagai auctor che assolve differenti funzioni.

In primo luogo, attua come voce autoritaria e critica rispetto al poema, una voce cosciente del suo ruolo nell'appropriazione e ricreazione della Commedia in base al proprio gusto, al contesto in cui opera e ai condizionamenti stessi del medium impiegato. Si tratta di una presenza che è sia auctoritas, in quanto proiezione dell'autore che ha selezionato, adottato, adattato, trasformato, ridotto e amplificato non solo l'ipotesto, ma anche il complesso reticolato intertestuale che da esso deriva; sia mediatrice transtemporale e transculturale tra la Commedia e il lettore9 moderno, filtrandone gli anacronismi e le divergenze culturali. Per questa ragione la voce del Dante auctor, cosciente della responsabilità verso la forma e lo stile della propria opera, viene rimossa: egli non è, ovviamente e necessariamente, l'autore del manga.

In secondo luogo, ma evidente conseguenza del primo punto, è la predilezione di Nagai per il livello narrativo dell'opera e per il senso letterale piuttosto che quello allegorico. Il mangaka sceglie di adattare quei versi carichi d'azione e di pathos che, (ri)combinati con le atmosfere e le suggestioni delle tavole di

8. Non vogliamo entrare in questo contesto nel dibattito sulla validità della divisione binaria Dante auctor/Dante agens che esula dai propositi di questo articolo. Ciononostante, avalliamo le questioni sollevate, tra gli altri, da Maria Gabriella Riccobono, per la quale né l'uno né l'altro sono responsabili della narrazione, esistendo nel mezzo un terzo io "al quale è eminentemente dato il compito di narrare il passato, i casi occorsi durante il viaggio", (Riccobono 20I3: 9 e I7-18).

9. Intendiamo qui un ipotetico lettore medio giapponese a cui il manga è rivolto. 
Doré hanno contribuito a creare il suo immaginario. Non trova posto (né può trovarne) in Dante Shinkyoku "quel gusto medievale per il sovrasenso e per la significazione indiretta" (Eco 1987: I6I), ovvero tutta l'architettura simbolicoallegorica e l'indottrinamento teologico che sorregge la Commedia. Per questo, gli ammonimenti del Dante auctor che invitano il lettore a riconoscere "la dottrina che s'asconde sotto il velame de li versi strani” (If. IX vv. 62-63) vengono eliminati, così come vengono rimosse similitudini e analogie. Ne consegue che spesso il Nagai auctor intervenga per esplicitare un'allegoria scegliendo la via della spiegazione didascalica e dell'univocità interpretativa. Così, le tre fiere e la selva oscura vengono risolte in una didascalia, davanti allo smarrimento di Dante: "la lonza rappresenta la lussuria e la mancanza d'integrità; il leone la violenza e il potere; la lupa la cupidigia e la cospirazione; infine la selva oscura è la profondità dei peccati." (V. I, p. I9). Allo stesso modo, quando Dante si trova a cospetto dell'angelo guardiano ( $P g$. IX vv. 94-IO2) un'altra didascalia spiega il significato dei colori dei tre scalini: “[...] In quest'ordine essi indicano la sincera confessione dei propri peccati commessi (bianco), il pentimento (indaco) e la felicità che ne deriva come ricompensa (rosso)" in cui possiamo osservare licenze sia nell'ipotesi interpretativa che nell'indicazione dei colori.

Ed ancora si svela l'allegoria dei colori nelle tre donne danzanti della processione nel Paradiso Terrestre (Pg. XXIX vv. I2I-I29). Questa volta è proprio Dante a palesarla senza alcuna incertezza: "Giusto, comprendo l'allegoria di quei colori!" E nella vignetta successiva: "Indicano l'amore, la speranza e la fede" (V. II, p. 330), senza però menzionare che si tratta delle virtù teologali.

Quest'appiattimento che non lascia spazio alla speculazione ermeneutica assolve anche una funzione didattica. Lo stesso Nagai rivela, nel commento che accompagna il manga, che ha dovuto compensare con inserti esplicativi alcune parti che altrimenti sarebbero risultate incomprensibili ad un lettore moderno giapponese (Nagai 1998b: 362). Questo tipo d'interventi sono tutt'altro che avulsi dal mondo del manga che proprio verso la fine degli anni ottanta iniziò a sviluppare un genere proprio didattico-educativo (Bouvard 20II). Lo stesso Nagai pubblicò nel 2000 una versione della Commedia come parte di un "Corso di letteratura mondiale" edito da Kodansha (永井豪の世 界文学講座 ダンテ『神曲』).

Ciò detto, gli interventi del Nagai auctor, si osservano soprattutto nell'adattamento dell'Inferno, si riducono drasticamente nel Purgatorio e sono totalmente assenti nel Paradiso. In quanto al contenuto, ampliano o forniscono informazioni storiche, biografiche e allegoriche di personaggi o episodi. In quanto a ubicazione e forma, adottano caratteristiche fluide; il testo esplicativo può apparire con o senza cornice, a destra o nella parte superiore di una vignetta, in uno spazio extradiegetico dedicato, oppure invadendo lo spazio diegetico. Se l'esilio di Dante da Firenze e l'incontro tra il poeta e Beatrice 
estratto dalla Vita Nuova si inseriscono, come abbiamo analizzato, nel meccanismo narrativo, puramente chiarificatori sono invece gli interventi che descrivono l'origine del Minotauro (attraverso due tavole che risolvono il mito di Pasifae e quello di Teseo e Arianna, Vol. I, pp. 265-266); l'origine dei Centauri; la topografia dell'Inferno e del Purgatorio; le biografie storico-mitologiche di Virgilio, Flegias, Perseo, Farinata degli Uberti, Achille, Pier delle Vigne, Bertrand de Born e Crono. Gli interventi più brevi e "sottili" riguardano le definizioni del Purgatorio (Vol. I, p. 38) e dell'arca dell'alleanza (Vol. II, p. 225), collocati addirittura nel margine esterno. ${ }^{\mathrm{IO}}$

I rimanenti interventi adottano spesso uno stesso formato: il testo è collocato nella parte superiore della tavola, senza riquadro e all'interno di una vignetta unica, separata dal contesto diegetico (ad esempio attraverso un cambio radicale dello sfondo). Fanno eccezione gli interventi su Virgilio, Pier delle Vigne e Bertrand de Born. Per quanto riguarda Virgilio, il testo biografico è collocato a destra in una vignetta "fluttuante" senza bordi (riservata in genere ai momenti di approfondimento psicologico dei personaggi) posta al centro della tavola e senza interrompere la diegesi. Inoltre, l'allineamento del testo con l'immagine (un primo piano di Virgilio che parla) colloca entrambi sullo stesso livello di significazione rispetto a quando il testo occupa la parte superiore (Vol. I, p. 22). A Pier delle Vigne vengono riservate due vignette che combinano le due posizioni del testo, che in una è collocato nella parte superiore e nell'altra a destra (Vol. I, pp. 290-29I). A Bertrand de Born è invece dedicata solo una didascalia fluttuante su sfondo bianco, senza immagine dedicata (Vol. II, p. 47).

Un intervento del tutto singolare e unico riguarda invece il conte Ugolino. Attraverso una didascalia fluttuante su spazio bianco, il Nagai auctor si inserisce nella narrazione intradiegetica del conte chiedendosi se Dante abbia voluto raccontare le sue vicende, così come avviene per Francesca, a causa della loro contemporaneità e aggiunge: "Sarà dovuto al trauma per tale vicenda che Dante abbia voluto metterne in scena il protagonista?" (V. II, p. II6). È questa la voce di un lettore curioso, nettamente separata da quella di Dante, che converte questa didascalia apparentemente innocente in una prova tangibile della distanza tra una e l'altra.

\section{Procedimenti formali DELL'AdATTAMENTO}

In questa sezione prenderemo in esame alcuni degli elementi e delle tecniche impiegate da Nagai per realizzare l'adattamento. In primo luogo, analizzeremo le tavole o le vignette che adattano le illustrazioni di Gustave Doré,

IO. Nella versione italiana queste informazioni vengono inserite nel gutter (lo spazio bianco tra vignetta e vignetta). 
descrivendo quei procedimenti che permettono la loro integrazione nella continuità narrativa del manga. In secondo luogo, osserveremo in linea generale come agiscono i meccanismi di significazione del linguaggio proprio del manga nella trasposizione della Commedia, inserendo alcuni esempi di sequenze prive di riferimenti diretti a Doré. In entrambi i casi, ci soffermeremo sui tre aspetti del linguaggio grafico già menzionati: l'incorporazione di morfemi, il framing e la sequenzialità.

Per quanto riguarda gli adattamenti delle illustrazioni dell'incisore francese, le tecniche più usate consistono nell'inclusione di testo e di affissi, concretamente onomatopee e mimesi, linee cinetiche, stelle d'impatto. In una sola occasione viene impiegato il morfema di reduplicazione e in nessun caso si adottano morfemi di soppiantamento o sfondi soggettivi.

Onomatopee e mimesi vengono impiegate solo negli adattamenti delle illustrazioni dell' Inferno e del Purgatorio. Più in concreto, nell' Inferno vengono usate per denotare: lo scorrere dell'acqua dell'Acheronte (If. III vv. 82-83/ vol. I, pp. 66-7); il ronzio delle anime che volteggiano attorno a Paolo e Francesca (If. V v. 3I-32/vol. I, p. 98); il suono del Minotauro che si ritorce (If. XII v. II-2/vol. I, p. 269); il sibilo delle frecce dei centauri e le grida delle vittime (If. XII, v. 77-8/vol. I, p. 274); i lamenti di Pier delle Vigne (If. XIII v. 33/vol. I, p. 288); i gemiti di dolore, la rottura dei tronchi e i movimenti di fuga degli scialacquatori nel bosco dei suicidi (If. XIII v. II8/vol. I, p. 293); il ronzio nella ripa discoscesa dove dimora Gerione (If. XVII v. 7-8/vol. I, p. 306); lo schiocco delle sferze sopra i corpi dei ruffiani (If. XVIII v. 38/vol. I, p. 3I4); l'impatto di Ciampolo di Navarra con l'acqua (If. XXII v. I26/vol. I, p. 340); le grida di sofferenza attorno a Pier da Medicina (If. XXVIII v. 73-5/vol. II, p. 42); e il tormento dei falsari (If. XXX v. 32-3/vol. II, p. 58).

Nel Purgatorio invece, onomatopee e mimesi esprimono l'ira della folla inferocita mentre lapida Santo Stefano ( $P g . \mathrm{XV}$ v. I03/vol. II, p. 255); il respiro affannoso degli accidiosi ( $P g$. XVIII v. 87, vol. II, p. 280); le grida di sofferenza dei lussuriosi nel fuoco (Pg. XXV v. II7/ vol. II, p. 304).

In alcuni adattamenti l'uso di onomatopee e mimesi integra le immagini con il fine di rendere minuziosamente la trasposizione dei versi danteschi, specialmente laddove questi esprimano azioni e suoni. È il caso dell'illustrazione degli scialacquatori che fuggono (If. XIII v. II8/ vol. I, p. 293), dove i suoni delle concitate azioni descritte nei vv. II5-I26 vengono ripresi da onomatopee corrispondenti: la rottura di rami ed arbusti; i lamenti di dolore; il latrato delle "nere cagne" (Fig. 4).

Le linee cinetiche accompagnano gli adattamenti delle tre parti del manga e sono specialmente rilevanti nel Paradiso, dove illustrano la verticalità e trasmettono la sensazione di ascesa. In alcune illustrazioni dell' Inferno e del Purgatorio le linee cinetiche si abbinano agli effetti sonori per rendere meglio 
l'azione narrata nei versi danteschi. Ad esempio, nel già citato adattamento dell'incisione dedicata al Minotauro, le linee che descrivono i movimenti di Dante, Virgilio e del Minotauro, che si morde e si ritorce al cospetto dei due pellegrini, accompagnano il morfema di reduplicazione della testa del Minotauro e gli effetti sonori corrispondenti, conferendo grande vivacità, drammaticità e urgenza a tutta la vignetta che nell'originale di Doré appariva estremamente statica (Fig. 5).

Oltre ai morfemi visivi, un ulteriore intervento nella riproduzione delle litografie di Doré è l'inclusione di testo, che appare in differenti forme in base alla funzione che ottempera: all'interno di nuvolette di dialogo o monologo; come didascalia con o senza bordi, impiegata nelle tre parti del manga; e come titolo all'inizio di capitoli nelle pagine splash raccolte principalmente, ma non solo, nel Purgatorio.

Al di là della funzione propriamente verbale, l'inclusione del testo negli adattamenti di Doré serve anche da trait d'union con le vignette attigue, rinforzando altre tecniche utilizzate per integrare l'illustrazione nel flusso narrativo o per comunicare una certa atmosfera o sensazione. Una di queste tecniche consiste nel manipolare i margini della tavola o della vignetta dov' è contenuta la riproduzione. A volte la vignetta smargina, ovvero esce da uno, più o tutti i margini prefissati. Altre volte è un solo elemento (un personaggio o un oggetto) a fuoriuscire dai propri confini, accentuando la propria importanza o la propria imponenza sul resto della scena (Eisner 1985: 46). Attraverso questi stratagemmi il tempo e l'azione "scappano" dall'illustrazione adattata incorporandosi così, graficamente e simbolicamente, con il resto della narrazione. In questo senso operano anche le tecniche di ritaglio (crop) o di zoom dell'illustrazione, la manipolazione della posizione di alcuni personaggi o l'uso di inserti (inset panels), di cui presentiamo i tre casi adottati.

Il primo inserto viene utilizzato nell'adattamento della litografia If. XXXII vv. 98-99 (vol. II, pp. I08-9), inserendo sotto il margine superiore destro una vignetta che mostra il primo piano di Bocca degli Abati mentre interpella Dante, focalizzando così l'attenzione del lettore sul dialogo tra i due personaggi. Il secondo caso si osserva nell'adattamento dell'illustrazione If. XXXIV vv. I33-I34 (vol. I, p. I45) con un doppio inserto: il primo "invade" l'immagine a destra con due primissimi piani di Virgilio e Dante che mostrano le loro espressioni di felicità per essere usciti dall' Inferno; il secondo inserto, collocato nella parte inferiore sinistra, mostra un primo piano di Dante che piange di allegria. Infine, l'illustrazione $P g$. II v. 42 (vol. I, p. I47) è adattata con un inserto di un primo piano di Beatrice che smargina la vignetta stessa.

In questi casi, la tecnica dell'inserto serve per mostrare la simultaneità delle azioni che avvengono nella stessa scena oltre a risaltare l'importanza dei personaggi e avvicinarli (letteralmente ed emotivamente) al lettore. Negli ul- 
timi due casi descritti inoltre, l'inserto intensifica la tensione del momento poiché coincide con il climax della trama: l'uscita dall'inferno nel primo caso e l'incontro con Beatrice nel secondo. Significativamente questi due inserti coincidono anche con una brusca alterazione degli eventi rispetto ai versi della Commedia. All'uscita dall'inferno infatti, il Dante di Nagai anziché "riveder le stelle", è accolto dalla luce del sole. Subito dopo, Nagai chiude l'Inferno con l'illustrazione che Doré realizzò per Pg. II v. 42, sostituendo l'apparizione dell'angelo nocchiero con Beatrice (Fig. 6). Dato che il Purgatorio di Nagai si apre immediatamente con l'incontro di Dante e Virgilio con Catone potremmo speculare sul fatto che il mangaka avesse pensato inizialmente di adattare solo la prima cantica, chiudendola con l'incontro di Dante con l'amata Beatrice, che di fatto, non tornerà ad apparire fino al Paradiso.

In linea generale, gli interventi più cospicui si osservano nell'adattamento dell'Inferno, denotando forse una maggiore attività interpretativa e creativa da parte di Nagai. Infatti, una volta riprodotte, le illustrazioni di Doré vengono anche utilizzate per ricreare, nelle vignette successive, il mondo che contengono, attraverso operazioni di manipolazione e scomposizione. È come se una cinepresa entrasse nel mondo creato da Doré e ne catturasse diversi frammenti e piani mettendoli a fuoco da differenti prospettive. In questo modo, le illustrazioni si tendono e si svolgono per riempire gli spazi narrativi tra una e l'altra, integrandole con la trama estratta dai versi danteschi. Spesso quest'operazione ristabilisce anche gli elementi ritmici, l'azione e l'atmosfera dei versi che si perdono o sfumano nella staticità delle litografie di Doré.

Osserviamo ad esempio l'adattamento dell'illustrazione If. XIII v.Io (vol. I, p. 285) in cui Dante e Virgilio entrano nel bosco dei suicidi. La riproduzione di Nagai non modifica nulla dell'illustrazione originale, così, nel silenzio del paesaggio vediamo le piccole silhouettes dei due pellegrini e le arpie annidate sugli alberi. Tuttavia, subito dopo, gli eventi incalzano attraverso una scomposizione dell'illustrazione in differenti vignette che creano un'atmosfera inquietante. Mediante l'uso cospicuo di effetti sonori e linee cinetiche, le arpie si animano in tutta la loro violenza, strillando e appigliandosi agli alberi coi loro artigli, traducendo con grande efficacia i versi corrispondenti (Ali hanno late, e colli e visi umani/piè con artigli, e pennuto 'l gran ventre;/fanno lamenti in su li alberi strani. If. XIII vv.I3-I5.) e potenziando la concitazione di una sequenza che culminerà con Dante che spezza il ramo dal corpo/albero di Pier delle Vigne.

Un altro esempio significativo di questa tecnica di riempimento tra una litografia e l'altra è mostrato nell'adattamento del canto VIII dell' Inferno. Nagai ricalca senza modifiche le tre illustrazioni che Doré realizzò per il canto (vv. 29-30/vol. I, p. I90; 4I-42/ vol. I, p. 194; I09-IIo/ vol. I, pp. 200-I). Queste stesse immagini vengono poi frammentate e rielaborate per narrare tre scene 
principali: l'incontro con Flegias, vv I-30; la conversazione con Filippo Argenti, vv. 67-I30; l'arrivo alla città di Dite, vv-67-I30 (Fig. 7, 8, 9).

Nella prima scena la cinepresa di Nagai gira attorno alla barca di Flegias, partendo da uno spettacolare piano zenitale inserito in una vignetta verticale in cui Flegias, Dante e Virgilio sono riprodotti con postura e gesti uguali a quelli nell'illustrazione di Doré. Successivamente, attraverso transizioni "da soggetto a soggetto", l'illustrazione viene frammentata mostrando vari dettagli dell'azione che accadono simultaneamente all'interno della stessa scena. In concreto, si mostrano il primissimo piano di Dante e dei diversi condannati nell'acqua. Seguono altre rapide transizioni che alternano piani ravvicinati della barca mentre fende l'acqua o mentre investe le anime dannate.

Nella seconda scena, la cinepresa si concentra sulla figura di Filippo Argenti aggrappato alla barca, ritratto con primi e primissimi piani mentre dialoga con Dante. Nella terza scena, la cinepresa scompone l'ultima illustrazione di Doré creando campi e controcampi tra la barca e la riva della città di Dite. Una ripresa in soggettiva mostra la città dal punto di vista della barca; poi la prospettiva si capovolge, mostrando la barca in lontananza vista dalla riva.

Va sottolineato come non esista una regolarità né nell'adattamento né nella scomposizione delle illustrazioni di Doré. Se nella parte iniziale dell'Inferno Nagai preferisce "interpretare" le illustrazioni attraverso la frammentazione e la ricomposizione, a partire dal capitolo "Il quinto cerchio" (corrispondente al Canto VIII) la riproduzione delle litografie è quasi totale, mentre la tecnica di scomposizione è dedicata a riempire lo spazio narrativo tra un'illustrazione e l'altra, come mostrato negli esempi anteriori. Inevitabilmente, tanto una tecnica come l'altra permeano stilisticamente tutto il manga di Nagai dotandolo di una chiara impronta doreana, evidente nella caratterizzazione di alcuni personaggi, nella descrizione visiva delle pene o nella rappresentazione del paesaggio dotato di una forte carica espressiva/espressionista. Eppure, su questa impronta Nagai agisce inserendo nei personaggi e nei paesaggi tardoromantici elementi propri non solo del manga ma della cultura giapponese, facendo di Dante Shinkyoku una specie di pastiche estetico.

Riscontriamo un'evidente ispirazione all'iconografia buddista dei rotoli giapponesi e delle maschere del teatro Noh, ad esempio nella rappresentazione del viso di Caronte (vol. I, p. 64), Flegias (vol. I, p. 185) e nei volti degli iracondi del Purgatorio (vol. II, p. 252), con la quale si stabilisce una relazione tra i due mondi già elaborata da Kanzō Uchimura e Hakuchō Masamune e rilevabile anche nella terminologia utilizzata dai traduttori giapponesi della Commedia, più prossima alle rappresentazioni iconografiche buddiste che cristiane (Hirakawa 1996: 50).

Senza dimenticare i motivi visivi propri delle creazioni di Nagai che, come summenzionato, sono a loro volta frutto di un'influenza di Dante, Doré e 
della tradizione classica greco-romana e che ritornano, in un certo modo, a far parte di Dante Shinkyoku, particolarmente nei personaggi grotteschi, nei mostri e negli esseri ibridi.

Nel momento in cui Dante Shinkyoku viene pubblicato, il pubblico di Nagai è già avvezzo alla gorgone Medusa di Mao Dante, all'arpia Silene di Devilman o ai centauri come Teotras di Mazinger, Kentol $\Gamma_{7}$ di Mazinger $Z_{0}$ il cavallo Antares che permette a Jeeg di convertirsi in un centauro. Nagai ha già disegnato il Conte Blocken con la testa separata dal tronco e il ministro Argos e il generale Ardias che esibiscono una testa al posto della mano sinistra, realizzati con un'estetica che ricorda il Bertran de Born di Doré (Fig. Io); l'esercito di rettili umanoidi comandati dal generale Dreidow richiama la metamorfosi di Agnello Brunelleschi; l'imperatore delle Tenebre, il generale Nero o il barone Ashura, che possiedono corpi dove convivono vari volti, evocano il Lucifero Tricipite, "lo'mperador del doloroso regno"; la marchesa Yanus ha una doppia testa rotante ispirata al dio romano omonimo, ma anche agli indovini danteschi; e i mecha, i giganti meccanici, inevitabilmente ci riportano ai giganti del Canto XXXI dell'Inferno.

Per quanto riguarda i meccanismi di significazione, osserviamo come gli elementi formali che compongono il linguaggio del manga (morfemi, framing e sequenzialità) variano al variare dei temi, dell'atmosfera, delle emozioni e delle questioni che si vogliono esprimere nelle diverse parti dell'adattamento.

Nell'Inferno e in certa misura anche nel Purgatorio, dominano le scene d'azione e di violenza che si alternano a scene di dialogo e di confronto verbale su questioni etiche e morali. È inoltre presente una certa dose di erotismo, un altro tema caro a Nagai, che approfitta di ogni apparizione di personaggi femminili per includere nudi in pose provocanti e sensuali. Si tratta di tematiche proprie dei generi shōnen, seinen e seijin, diretti rispettivamente a un pubblico maschile adolescente, giovane e adulto, ed adottano, salvo qualche eccezione, le rappresentazioni visive corrispondenti a questi generi. Nel Paradiso invece, predominano il tema romantico e l'atmosfera mistica e contemplativa con il conseguente impiego di una rappresentazione formale propria del shōjo romantico, rivolto, in generale, a un pubblico femminile adolescente.

L'analisi del framing mostra come le tematiche trattate nelle sequenze dell'Inferno e del Purgatorio vengano accompagnate da vignette dai bordi asimmetrici, spigolosi e irregolari che aiutano a trasmettere il tumulto emotivo e psicologico dei personaggi o la vivacità dell'azione. Concretamente, le scene più violente impiegano vignette triangolari, acute, affilate e allungate che illustrano efficacemente la tensione tra movimento ed emozione. Nell' Inferno, le forme oblique delle vignette servono anche a comunicare l'asprezza dei paesaggi dantesco-doreani, fatti di precipizi e voragini abissali attraverso i quali i personaggi si arrampicano o si calano. Predominano inoltre nell' $I n-$ 
ferno e nel Purgatorio le vignette di piccole dimensioni, con un conseguente aumento del numero medio di vignette per tavola che conferiscono ritmo e velocità alle sequenze d'azione e permettono un gioco di contrasti repentini con vignette grandi, a tavola intera o a doppia tavola, creando una sensazione di insicurezza e spaesamento. Numerose vignette poi oltrepassano i limiti della propria tavola sconfinando nella successiva, o smarginano occupando tutta la pagina, creando un effetto di "spazio eterno" conforme all'ambiente rappresentato (McCloud I994: I09).

Degno di nota è il framing delle vignette all'interno di una tavola che evoca la composizione dei kanjis, situando nella parte destra una vignetta grande che rappresenta il radicale e nella parte sinistra una vignetta più piccola che affina il senso della narrazione. ${ }^{\text {II }} \mathrm{Ci}$ si appella così al tipo di "kanji gigante" che secondo Jean-Marie Bouissou permette ai lettori che conoscono la lingua giapponese di "leggere" le pagine del manga come se si trattasse di un ideogramma (Bouissou 2OII).

Rispetto a Inferno e Purgatorio, nel Paradiso si impiegano meno vignette per tavola, con un predominio delle illustrazioni a doppia pagina smarginate, di cui abbiamo visto le proprietà connotative di atemporalità, che eliminano inoltre l'aggressività degli angoli. In questo modo, la forma delle vignette risulta più regolare, il che percettivamente rende invisibile il framing della tavola stessa (Bryce 20IO: I43). Come suggerisce Fusanosuke, i bordi delle vignette esprimono e stabiliscono l'ordine della struttura (Fusanosuke 2008: 70), di conseguenza la regolarità delle vignette faciliterà la lettura e trasmetterà un maggiore senso di armonia e serenità. In generale, nel Paradiso si conforma un ampliamento dello spazio e uno scorrere più lento dell'azione, creando paesaggi visivi e narrativi più lenti e tranquilli, che invitano alla contemplazione.

Riguardo ai morfemi visivi, Inferno e Purgatorio adottano in larga misura, come è da aspettarsi, quelli che trasmettono tensione, sofferenza, tenebrosità, sorpresa e azione. Affissi come la goccia di sudore, le lacrime e le linee deittiche (che convergono sul personaggio per esprimere tensione emotiva) mostrano angoscia, paura e sofferenza. La sorpresa è rappresentata, come nel fumetto occidentale, dalle metonimie "?" e "!", o con affissi come la scintilla attorno al viso, la stella scopica, o mediante morfemi di soppiantamento, come gli occhi vuoti o senza pupilla. Le fiamme che soppiantano le pupille esprimono invece crudeltà, ira e odio. Inoltre, la tenebrosità dell'anima viene trasmessa spesso attraverso l'uso di sfondi espressionisti, dominati dal nero o mediante la creazione di motivi di linee verticali scure e spesse, e contrasti accentuati. Le scene d'azione includono piani soggettivi che aumentano l'enfasi e la partecipazione del lettore, morfemi di reduplicazione e linee cinetiche e di zoom, tra le quali

II. Questo metodo di composizione viene spesso impiegato nel manga. A modo di esempio vedi Vol. I, pp. I64, I66, I68, I7I, I79. 
vanno menzionate specialmente le linee soggettive applicate al fondo, tipiche del manga, anziché al personaggio. Nel Purgatorio coesistono paesaggi aspri e scoscesi con ambientazioni bucoliche; dominano le tonalità grigie e ai morfemi tipici dell'Inferno si aggiungono elementi meno tetri, come le nuvolette bordate di note musicali.

Ma anche l'Inferno in alcuni casi eccezionali, legati alla narrazione di ricordi o visioni, rifugge le atmosfere tenebrose.

Per narrare le vicende di Paolo e Francesca, ad esempio, Nagai adotta un'ambientazione romantica, dominata dal bianco e i cui sfondi agiscono come rappresentazioni della soggettività di chi racconta: così, le mani unite dei due amanti si stagliano su un cuore grande; e il loro bacio è avvolto dalle fiamme, a significare la passione, ma preannunciando anche la tragedia imminente (Vol. I, pp. Io6-IIO). In altri casi, invece, ad interrompere l'atmosfera cupa dell'Inferno è qualche (raro) momento umoristico, provocato soprattutto dal contrasto tra la paura di Dante e la temperanza di Virgilio. Sottolineiamo inoltre le apparizioni di Beatrice nell' Inferno, cariche di erotismo e sensualità, accompagnate da elementi formali tipici di questa rappresentazione, come $\mathrm{i}$ bagliori e i riflessi che circondano la sua figura nuda, carnale e romantica al contempo. La Beatrice del Paradiso invece, vestita con una tunica bianca, circondata da luce e caratterizzata da un perenne sorriso innocente, abbandona ogni traccia di sensualità mostrandosi eterea e candida.

Il bianco è il colore del Paradiso, conferisce luce e leggerezza alle immagini, mentre linee cinetiche verticali accompagnano l'ascensione di Dante e Beatrice verso Dio. In questa direzione quindi agiscono anche i morfemi sottesi di felicità, luce, musicalità e ascesa: occhi brillanti o chiusi per esprimere allegria, lacrime di felicità, sfondi completamente bianchi o marcati da sottili linee deittiche, scintille e bagliori che circondano i personaggi come se fossero icone sante.

Purtroppo l'ampio divario tra il numero di tavole dedicate all' Inferno rispetto alle altre due parti, fa sì che queste risentano di una narrazione discontinua e frammentata. Intere porzioni delle due cantiche non vengono adattate e, soprattutto nel Paradiso, uno o più canti vengono addirittura condensati in una singola immagine, necessariamente allusiva o simbolica piuttosto che descrittiva. In altre occasioni, episodi distinti della Commedia vengono fusi in un'unica sequenza, con risultati comunque piuttosto pregevoli. Ė il caso ad esempio dell'immersione di Dante nel Lete ( $P g$. XXXI vv. 9I-I26) e dell'allegoria del carro ( $P g$. XXXII vv. I09-I6o), collocate tra l'altro nel Paradiso, che vengono risolte con un'unica sequenza visionaria senza testo e di grande forza evocativa (Fig. II). Ciononostante, il ridotto numero di tavole, il rifacimento pressoché identico delle illustrazioni di Doré prive di quel lavoro interpretativo-creativo che abbiamo apprezzato nell' Inferno, oltre all'assenza totale della 
voce esplicativa e autoritaria del Nagai auctor, fanno sospettare un minor interesse da parte dell'autore nell'adattare le due cantiche, e/o una certa fretta nell'esecuzione, che altrimenti avrebbe potuto estendere la capacità di rendere le loro qualità tonali e cromatiche (la musicalità, l'armonia, la luce) ben oltre i pochi esempi che ci rimangono.

Per esemplificare come i procedimenti formali finora descritti operino nel processo di trasmutazione dei versi danteschi, analizziamo ora una tavola (Fig. I2) che illustra la sequenza di Dante che esce dalla selva, qualche attimo prima di imbattersi nelle fiere, così come descritto in If. I, vv. 22-27. Nagai sostituisce l'analogia che apre la sequenza ("E come quei che con lena affannata..." ecc.) con le tre vignette superiori che mostrano Dante nel momento in cui sbuca fuori dalla selva. Si tratta di vignette piccole e oblique che enfatizzano l'azione e accompagnano il primo piano di Dante mentre corre, vivacizzato da linee cinetiche orizzontali e onomatopee che rappresentano la "lena affannata". La vignetta seguente mostra un dettaglio degli occhi di Dante con le immancabili gocce di sudore che catturano la nostra attenzione e la rivolgono verso il suo oggetto d'interesse, una stella nel cielo. Le altre tre vignette che compongono la tavola sono invece regolarmente rettangolari, denotando la fine della corsa e la momentanea sospensione dell'azione. La vignetta centrale, smarginata verso l'esterno della pagina, contiene un piano generale dell'ambiente in cui Dante è solo una silhouette nera stagliata nel cielo al bordo di un dirupo. La silhouette è accompagnata da linee cinetiche e onomatopee. Si tratta della trasposizione del verso "così l'animo mio, ch'ancor fuggiva" uno splendido verso già di per sé evocativo del topos dei cartoni animati dove l'anima del personaggio fugge dalla paura prima ancora che il corpo se ne renda conto. Nagai rende magistralmente la trasposizione fondendo il realismo della scena con un Dante che, trasformato in silhouette e accompagnato da linee cinetiche, perde la sua corporeità e continua idealmente a "fuggire". Infine, le due ultime vignette in fondo alla tavola mostrano due primi piani del poeta incupito che si volge indietro "... a rimirar lo passo/che non lasciò già mai persona viva”.

\section{Conclusioni}

Secondo quanto ha dichiarato lo stesso Go Nagai (1998b: 364), il traduttore della Commedia al giapponese (1989) Eriya Taniguchi un giorno gli disse: "Le culture si sovrappongono, devi provare a creare la tua Divina Commedia". Questa frase riassume il processo di adattazione realizzato con Dante Shinkyoku, che abbiamo analizzato in questo studio.

Dante Shinkyoku si converte quindi in un ulteriore strato di queste culture sovrapposte, ciò che abbiamo definito "il mondo Divina Commedia", che 
comprende i significati e le forme che il poema ha acquisito nel suo dialogo con tempi e spazi differenti, nel suo integrarsi ad ogni successivo presente.

Abbiamo osservato come l'esame degli elementi strutturali sia fondamentale per comprendere la natura "palinsestica" (Hutcheon 2006: 2I) di Dante Shinkyoku ed evidenziare le relazioni tra ipotesto e ipertesto attraverso cui si muovono le molteplici ramificazioni intertestuali del manga. A partire dalle illustrazioni di Gustave Doré, vera e propria ossatura dell'opera, Nagai intesse un eterogeneo assortimento di riferimenti e citazioni, dalla mitologia classica greco-romana alla pittura, dalla Vita Nuova a Milton, frutto di una ricerca non banale ed estemporanea dell'autore verso la cultura classica occidentale. Ma Dante Shinkyoku è anche il risultato di un movimento a spirale nel quale converge il "mondo Nagai", un mondo che è già sintesi transculturale e di cui la Commedia ha contribuito a modellare stilemi, temi e motivi visivi disseminati poi nelle opere del mangaka. Per questa ragione, quando il pubblico di Nagai approda a Dante Shinkyoku è in grado di percepire un'aria famigliare che riduce le distanze (temporali, culturali) con l'opera.

Abbiamo evidenziato poi, come il processo di appropriazione della Commedia da parte di Nagai venga "dichiarato" all'interno della struttura stessa del manga attraverso la sostituzione del Dante auctor con una nuova voce indeterminata ma non meno autoritaria. Si tratta innanzitutto di un'operazione necessaria di attualizzazione e transculturazione, giacché gli interventi del Dante auctor apparirebbero, nel nuovo contesto socio-culturale, non solo anacronistici, ma incomprensibili al lettore generico di manga. Inoltre la nuova voce esercita una funzione educativo-didattica mediante incursioni nella diegesi con incisi esplicativi su personaggi, figure mitologiche, luoghi, accadimenti storici ecc., che si sostituisce, per così dire, alla funzione di indottrinamento teologico del poema.

Infine ci siamo soffermati sull'analisi dei meccanismi di rappresentazione e significazione propri del linguaggio del manga in relazione alla trasmutazione intersemiotica, esaminando la funzione di morfemi visivi, framing e sequenzialità nel processo di transcodifica. Alcuni esempi presentati hanno illustrato l'operazione di "ecfrasi al contrario" attraverso cui il mangaka "traduce" l'informazione verbale in informazione visiva (Şerban 20I4: 22).

Per concludere, dobbiamo sottolineare che, sebbene siano già stati esaminati alcuni casi di adattamento di opere letterarie classiche al manga (ciò che alcuni autori definiscono manga-fying o mangafication), non è stata tuttavia ancora sviluppata una metodologia specifica per affrontare tale analisi. In questo senso, il nostro caso può essere considerato il punto di partenza di una proposta metodologica che coniughi gli studi manga con le teorie dell'adattamento. 


\section{BiBLIOGRAFIA}

Audeh, A., 2009, "Gustave Doré's Illustrations for Dante's Divine Comedy: Innovation, Influence, and Reception" in K. Fugelso (Ed.), Studies in Medievalism XVIII: Defining Medievalism(s) II, pp. I25-I64.

Auerbach, E., 2003, Mimesis: The Representation of Reality in Western Literature, Princeton, Princeton University Press.

Bouissou, J.M., 20IO, Histoire et univers de la bande dessinée japonaise, Arles, Picquier.

Bouvard, J., 20II, "Réflexions sur le manga éducatif", Image \& Narrative, I2. I, pp. I89-205.

Bryce, M., 2010, "A Look at Hikawa Kyoko's Kanata Kara", in Johnson-Woods, T., Manga: An anthology of Global and Cultural Perspectives, New York, Continuum Books, pp. I37-I56.

Capponcelli, L., 20I4, "Dal Paradiso all'Inferno: la Divina Commedia in Giappone attraverso Masamune Hakuchōe e Akutagawa Ryūnosuke”, Sturiale, in M., Traina, G. (Eds.), Parole e sconfinamenti. Studi offerti a Nunzio Zago per i suoi sessantacinque anni dai colleghi della Struttura didattica speciale di Lingue e letterature straniere di Ragusa, Leonforte, Euno Edizioni, pp. 73-85.

Cohn, N., 20I0, «Japanese Visual Language: The Structure of Manga», in JohnsonWoods, T. (Ed.), Manga: An Anthology of Global and Cultural Perspectives, New York, Continuum Books, pp. 187-203.

—, 2013, "Visual Narrative Structure", Cognitive Science, 37.3, pp. 413-452.

Danesi, A., 2016, "Romics 2016, intervista al grande Go Nagai", Movie for Kids, 5/05/2016, Milano (rev. 25 enero 2018) <http://www.movieforkids.it/news/ romics-20I6-intervista-al-grande-go-nagai/40537/>

Eco, U., 1987, Arte e belleza nell'estetica medievale, Milano, Bompiani.

—, 20I2, Dire quasi la stessa cosa. Esperienze di traduzione, Milano, Bompiani (ebook).

Eisner, W., 1985, Theory of Comics and Sequential Art, Florida, Poorhouse Press.

Fujimura, N., 2012, "Oto no nai oto o moji ni suru koto no jiremma: Manga ni okeru gitaigo-hyōgen no bunseki kara”, Bunka Kankyō Kenkyū, 6, pp. 62-71.

Fusanosuke, N., 2003, "Japanese Manga: Its Expression and Popularity», $A B D O n$ Line Magazine, 34. I (rev. 25 enero 2018) <http://www.accu.or.jp/appreb/og/pdf34I/34-IPoo3-005.pdf>

—, 2013, "Where Is Tezuka?: A Theory of Manga Expression", Mechademia, 8, pp. 89-107.

—, 2008, "Komatopia", Mechademia, 3, pp. 65-72.

Galbraith, P. W., 20I4, The Moé Manifesto: An Insider's Look at the Worlds of Manga, Anime, and Gaming, Clarendon, Tuttle Publishing.

Gō, I., 20II, "Tezuka Is Dead: Manga in Transformation and Its Dysfunctional Discourse", Mechademia, 6, pp. 69-82.

Hirakawa, S., 1996, "The Divine Comedy and the Nô Plays of Japan: an Attempt at a Reciprocal Elucidation", Comparative Literature Studies, 33, pp. 35-58.

Hutcheon, L., 2006, Theory of Adaptation, Londra, New York, Roudledge.

Ingulsrud, J. E., Allen, K., 2010, Reading Japan Cool: Patterns of Manga Literacy and Discourse, Plymouth, Lexington Books, pp. 95-I08.

Inoue, K., 2009, "Manga ni okeru nihongo onomatope no shin 'kōzō-hyōgen", Ósaka Daigaku Gengo Bunkagaku, I8, pp. 69-85.

Jones, A.M., 20I5, "Palimpsestuous" Attachments: Framing a Manga Theory of the Global Neo-Victorian", Neo-Victorian Studies, 8, I, pp.I7-47. 
Kinsella, S., 200o, Adult Manga: Culture and Power in Contemporary Japanese Society, Honolulu, University of Hawaii Press.

Kinsui, S., 2015, "Manga and Role Language Research", 7th International Scholarly Conference. Comicology: Probing Practical Scholarship, September 25-27, Kyoto, Kyoto International Manga Museum, Kyoto Seika University International Manga Research Center.

Lanier, D., 20I0, "Recent Shakespeare Adaptation and the Mutations of Cultural Capital", Shakespeare Studies, 38, pp. I04-II3.

Malone, P.M., 20I0, "From BRAVO to Animexx. de to Export: Capitalizing on German Boys' Love Fandom, Culturally, Socially and Economically”, in Levi, A., McHarry, M., Pagliassoti, D. (eds.), Boys' Love Manga: Essays on the Sexual Ambiguity and Cross-cultural Fandom of the Genre, Londra, Jefferson, McFarland \& Company Inc., pp. 23-43.

Mattalia, D. (a cura di), I975, Dante Alighieri, La divina commedia; I. Inferno, Milano, Biblioteca Universale Rizzoli.

McCloud, S., 1993, Understanding Comics: The Invisible Art, New York, Kitchen Sink Press for Harper Collins.

Miyake, L. K., 2008, "Graphically Speaking Manga Versions of The Tale of Genji”, Monumenta Nipponica, 63, 2.

Nagai, G., I998a, Mao Dante, Granaloro dell'Emilia, Dynamic Italia.

—, I998b, Dante Shinkyoku, Tōkyō, Kōdansha (2 vols.)

—, 20I4, La divina commedia, Milano, Edizioni BD (3 vols.).

Nakazawa, J., 2002, "Analysis of Manga (comic) Reading Processes: Manga Literacy and Eye Movement during Manga Reading", Manga Studies, 5, pp. 39-49.

Prisco, F., 2007, "Go Nagai, il padre di Goldrake: 'Devilman? E figlio del Lucifero di Dante", Il sole 24 ore del 27 aprile 2007 (rev. 25 genairo, 20I8), <http://www. ilsole24ore.com/art/SoleOnLine 4/Tempo\%2olibero\%2oe\%2oCultura/2007/o4/ intervista-nagai-prisco.shtml?refresh_ce=I>

Riccobono, M.G., 20I3, Dante poeta-profeta, pellegrino, autore, Roma, Aracne.

Rosenbaum, R., 20I2, Manga and the Representation of Japanese History, Londra, New York, Roudledge.

Scilabra, C., 2015, "Vivono fra noi. L'uso del classico come espressione di alterità nella produzione fumettistica giapponese”, Status Quaestionis, 8, pp. 92-IO9.

Şerban, A., 20I3, "Reinscribing sexuality: Manga Versions of Romeo and Juliet", Romanian Journal of English Studies, 9, pp. 335-350.

-, 20I4, "Manga-fying Shakespeare. You must explain. Tis fit we understand [it ]"(Hamlet IV. I.2), The European English Messenger, 23,I, pp. 2I-27.

Stam, R., 2000, "Beyond Fidelity: the Dialogics of Adaptation", in Naremore, J. (eds.) Film adaptation, New Brunswick, N.J., Rutgers University Press, pp. 54-76.

Taniguchi, E., (a cura di), 1989, Dante Alighieri, 神曲, Takarajimasha. 


\section{Morfologia Del LINGUAGGIO VISIVO GIAPPONESE}

Vol., pp. - Volume e pagina; A - Affisso; S - Soppiantamento; R - Reduplicazione; Sf - Sfondi; OU - occhio-umlaut

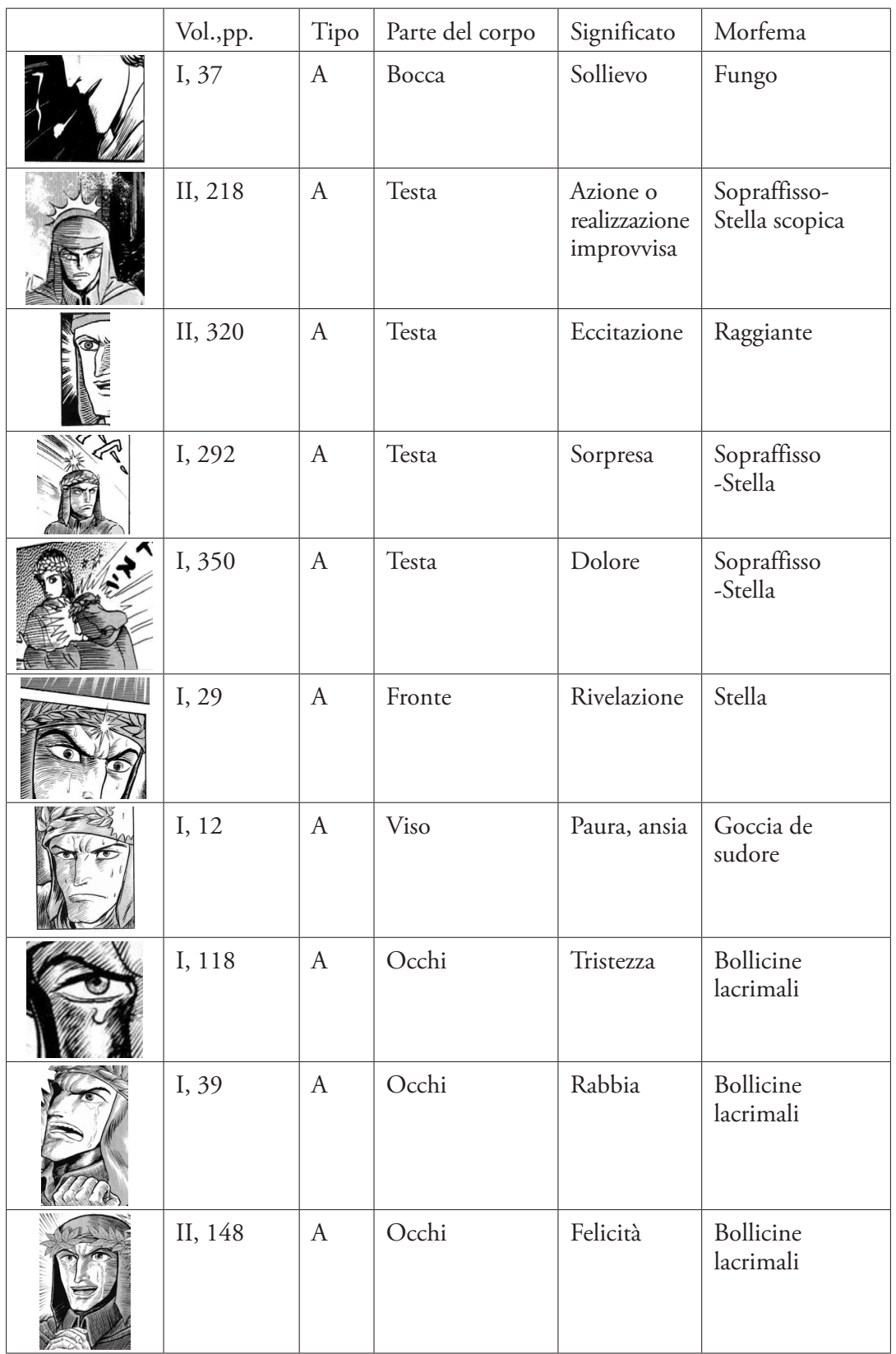




\begin{tabular}{|c|c|c|c|c|c|}
\hline & Vol.,pp. & Tipo & Parte del corpo & Significato & Morfema \\
\hline$r=-20$ & I, 12 & A & Testa & Sorpresa & Sopraffisso - ! \\
\hline & II, 166 & A & Testa & Stranezza & Sopraffisso - ? \\
\hline & II, 73 & A & Capo & $\begin{array}{l}\text { Sorpresa e } \\
\text { stranezza }\end{array}$ & Sopraffisso -!? \\
\hline & II, 170 & A & Capo & Ammutolito & Sopraffisso-...... \\
\hline & I, 352 & A & Lingua & Vertigini & Lingua fuori \\
\hline 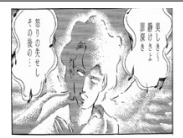 & II, 269 & A & & $\begin{array}{l}\text { Suono della } \\
\text { musica o } \\
\text { canzone }\end{array}$ & $\begin{array}{l}\text { Vettore-nuvoletta } \\
\text { musicale }\end{array}$ \\
\hline & I, 19 & $S$ & Occhi & Rabbia & Fiamma \\
\hline & I, 37 & OU & Occhi & $\begin{array}{l}\text { Dolore, } \\
\text { incredulità }\end{array}$ & Senza pupilla \\
\hline 11. & II, 142 & $\mathrm{R}$ & Corpo & $\begin{array}{l}\text { Emozione } \\
\text { conflittuale }\end{array}$ & Diversi corpi \\
\hline Lex & I, 267 & $\mathrm{R}$ & Testa & $\begin{array}{l}\text { Emozione } \\
\text { conflittuale }\end{array}$ & Testa triplicata \\
\hline
\end{tabular}




\begin{tabular}{|c|c|c|c|c|c|}
\hline & Vol.,pp. & Tipo & Parte del corpo & Significato & Morfema \\
\hline 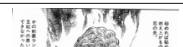 & I, 113 & SF & & Passione & Fiamma \\
\hline & I, 114 & SF & & \begin{tabular}{|l|} 
Sognando, \\
fantasticando
\end{tabular} & Cerchi sfuocati \\
\hline & I, 107 & SF & & Amore & Cuore \\
\hline & I, 41 & SF & & $\begin{array}{l}\text { Sublime etereo } \\
\text { intangibile }\end{array}$ & Linee ondulate \\
\hline
\end{tabular}

Tav. I. Nagai, G., I998, Dante Shinkyoku, Tōkyō, Kōdansha, vol. I e II. 

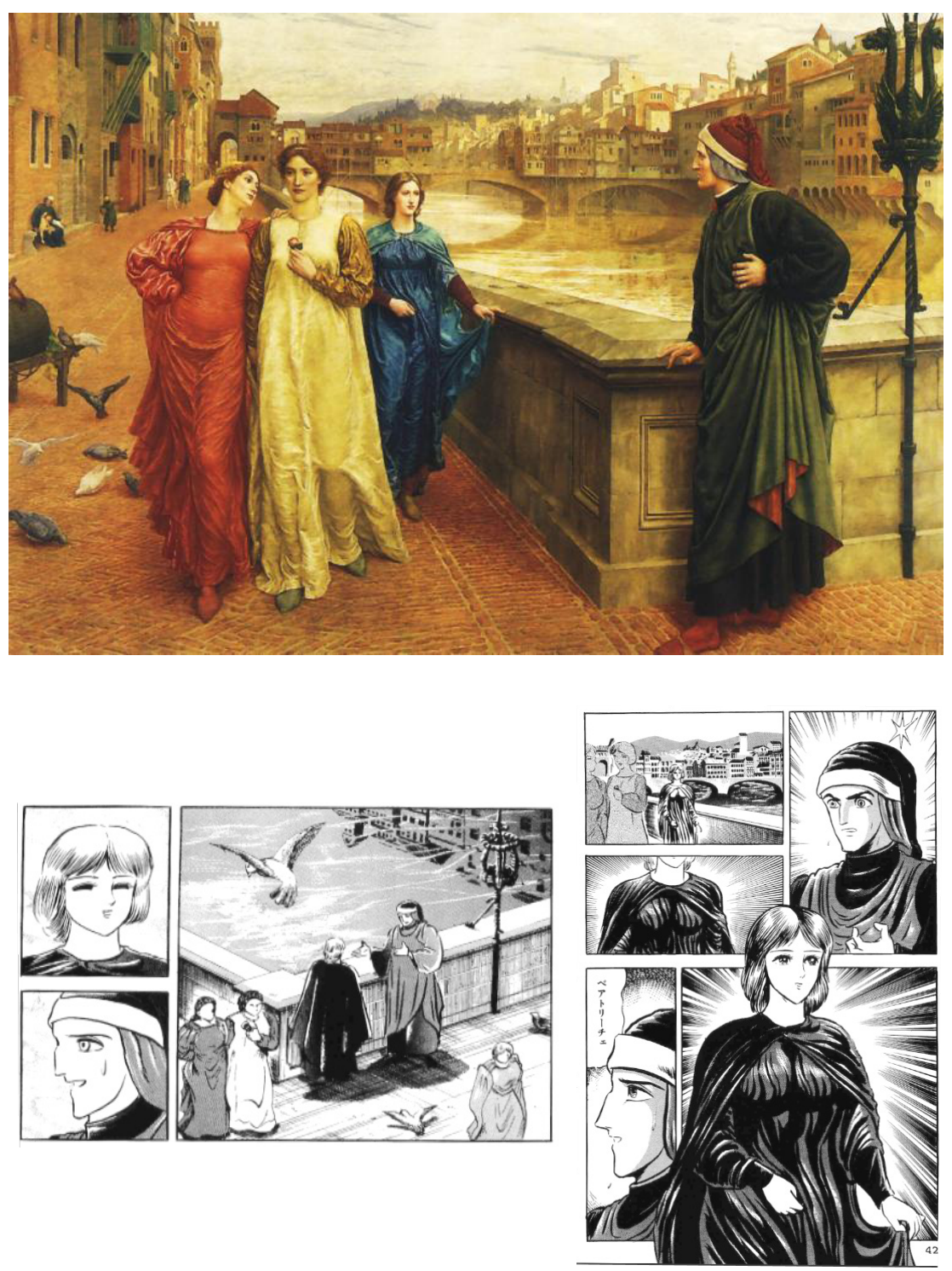

Fig. I. Henry Holiday, Dante and Beatrice, I883, olio su tela, Liverpool, National Museums and Galleries on Merseyside; Dante Shinkyoku, vol. I, pp. 42-43. 

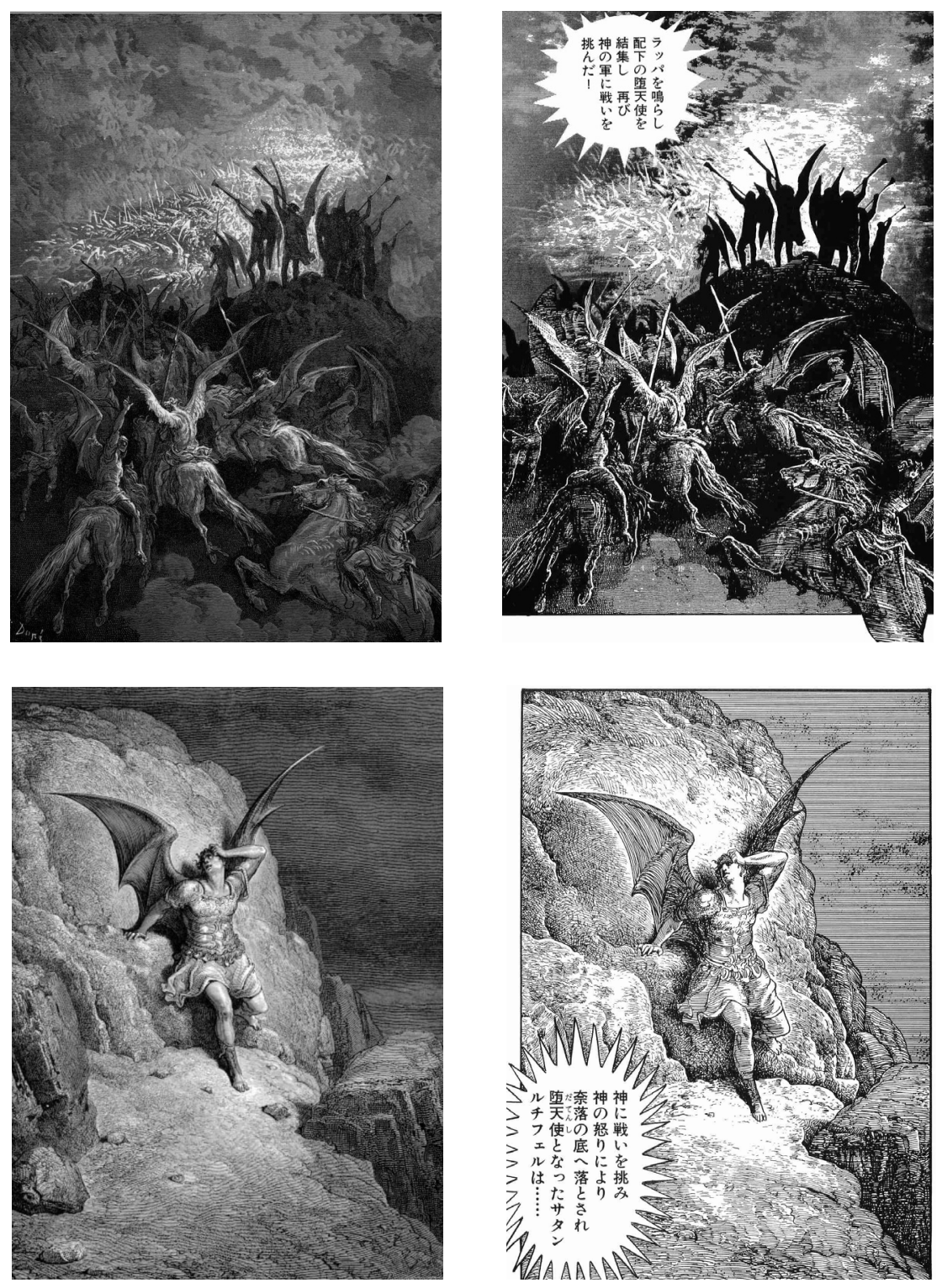

Fig. 2. Gustave Doré, I866, litografie 5, I3, I2 e 24, Milton, J., 2014 (I667), Paradise Lost, The University of Adelaide Library; Nagai, G., I998, Dante Shinkyoku, Tōkyō, Kōdansha, vol. II, pp. I30-I33. 


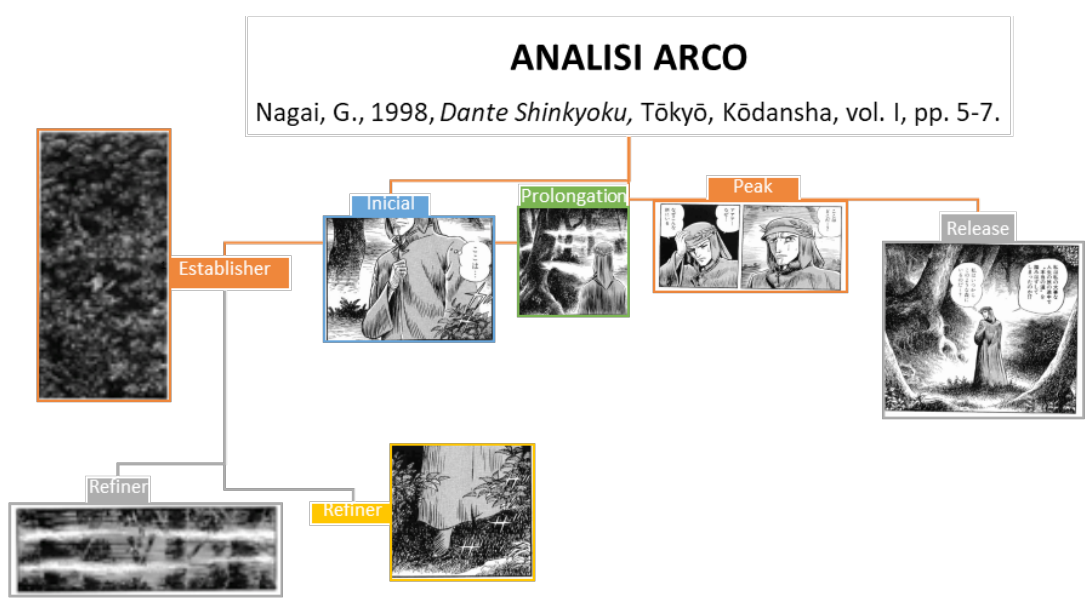

Fig. 3. Nagai, G., I998, Dante Shinkyoku, Tōkyō, Kōdansha, vol. I, pp. 5-7 

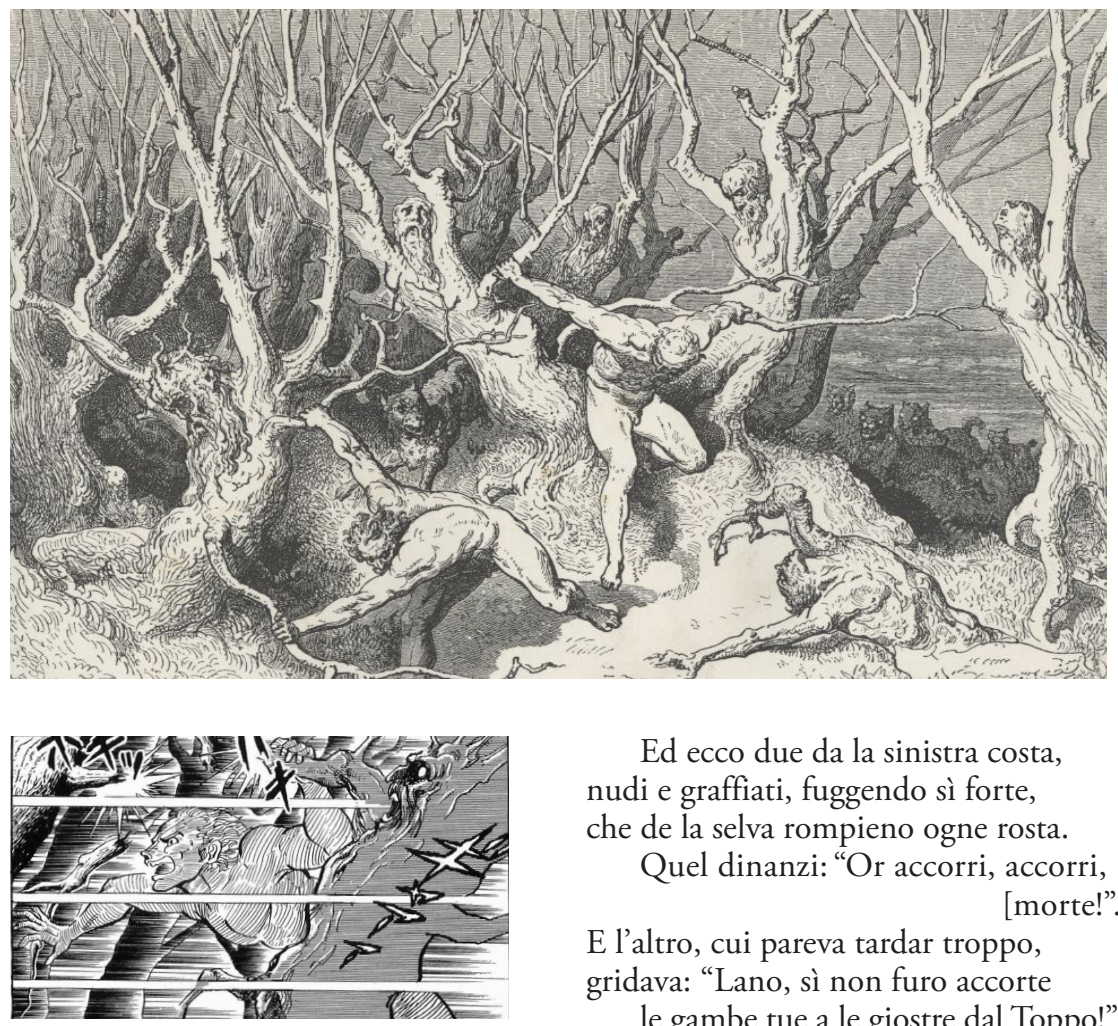

Ed ecco due da la sinistra costa, nudi e graffiati, fuggendo sì forte, che de la selva rompieno ogne rosta.

Quel dinanzi: "Or accorri, accorri, [morte!".

E l'altro, cui pareva tardar troppo, gridava: "Lano, sì non furo accorte le gambe tue a le giostre dal Toppo!”.

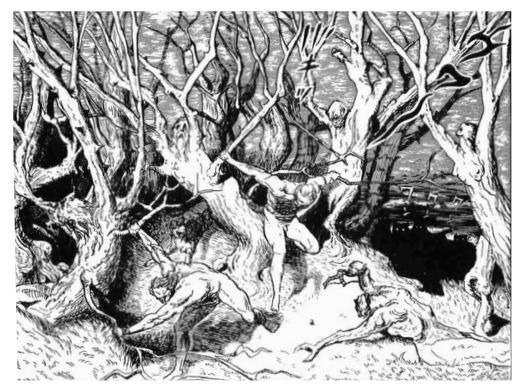
E poi che forse li fallia la lena, di sé e d'un cespuglio fece un groppo.

Di rietro a loro era la selva piena di nere cagne, bramose e correnti come veltri ch'uscisser di catena.

Fig. 4. Gustave Doré, Gli scialacquatori, If. XIII v. II8; Dante Shinkyoku, vol. I, p. 293; If. XIII vv. II5-I26. 

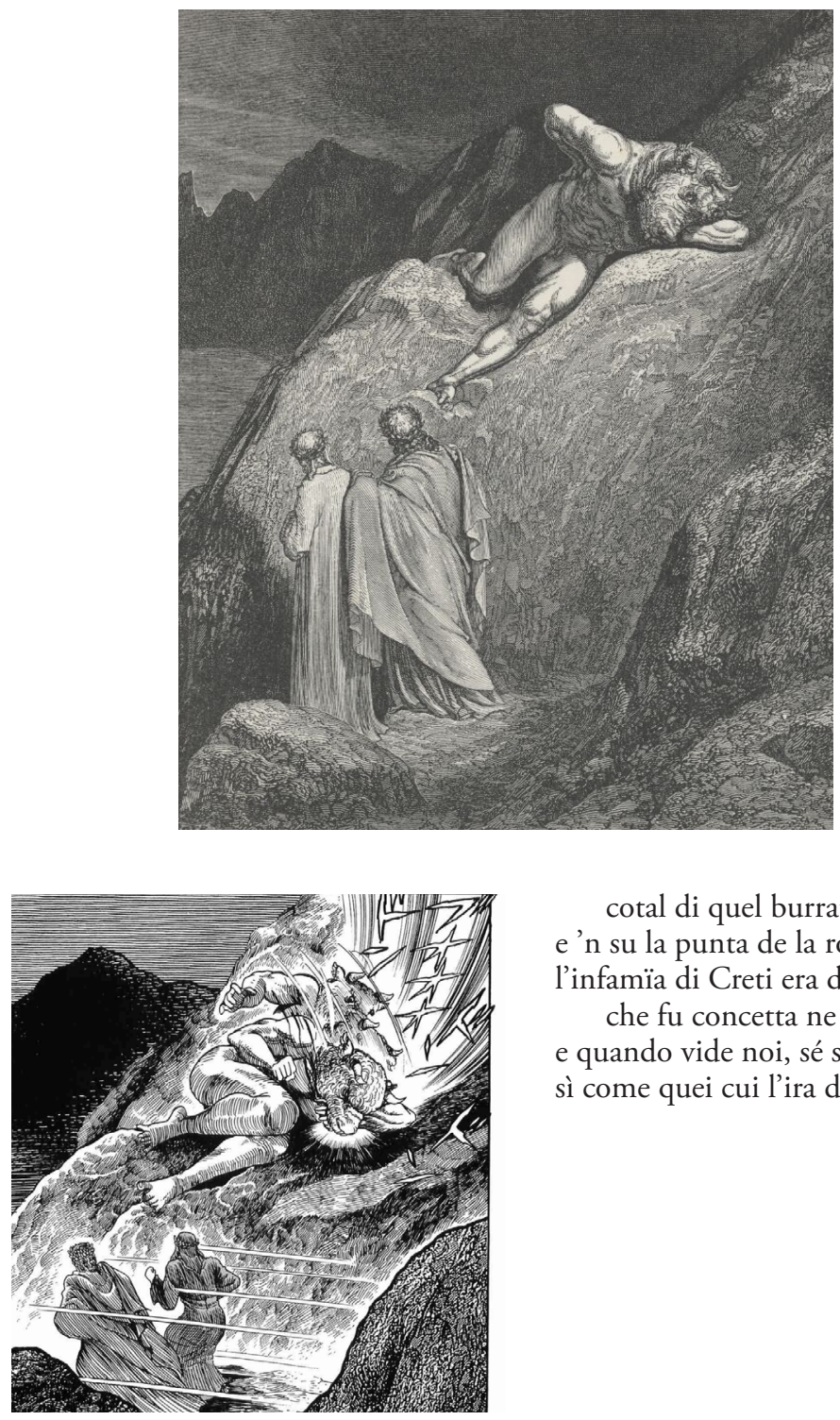

cotal di quel burrato era la scesa; e 'n su la punta de la rotta lacca l'infamïa di Creti era distesa. che fu concetta ne la falsa vacca; e quando vide noi, sé stesso morse, sì come quei cui l'ira dentro fiacca.

Fig. 5. Gustave Doré, Il minotauro, If. XII vv.II-I2; Dante Shinkyoku, vol. I, p. 267; If. XII vv. IO-I5. 


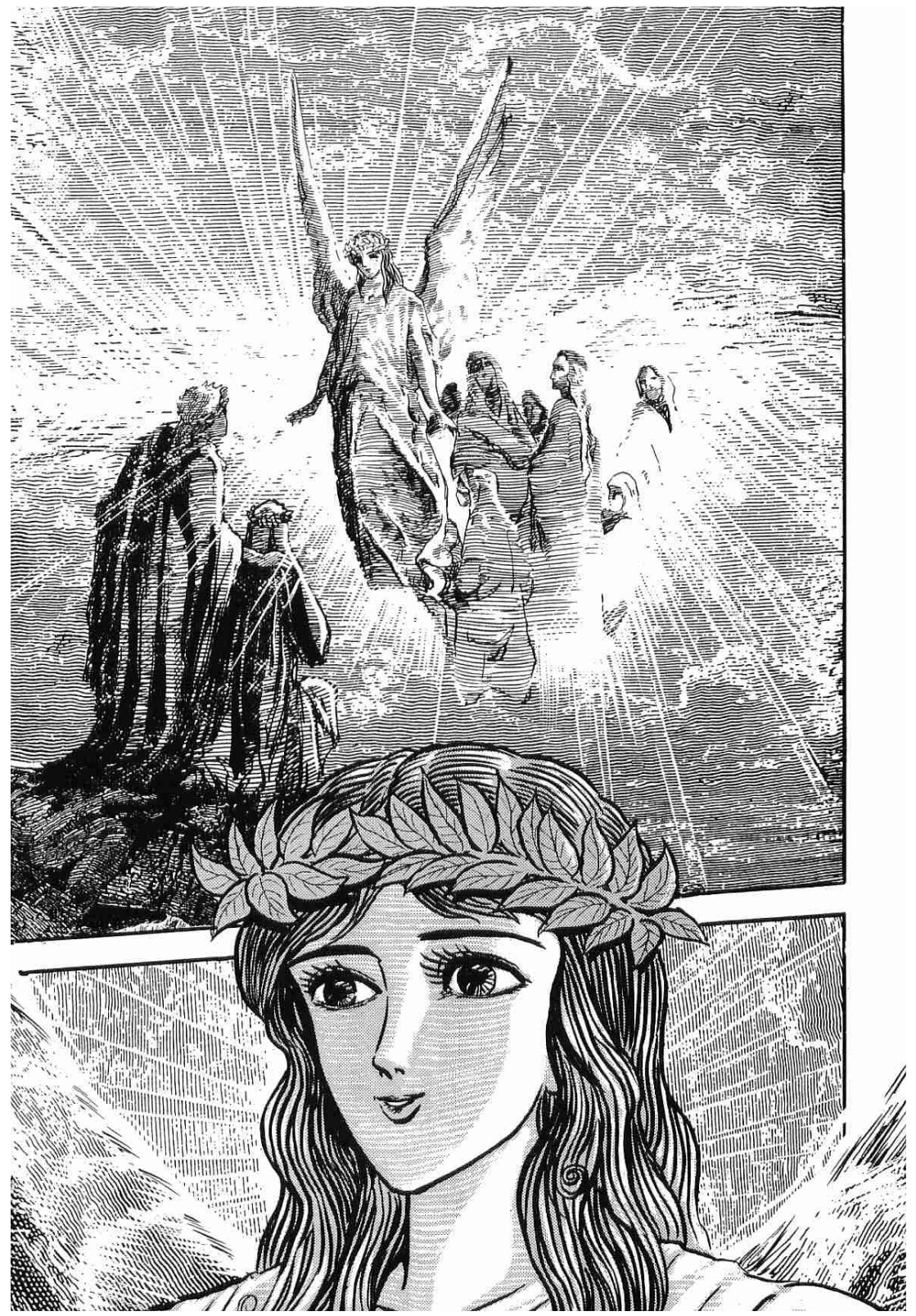

Fig. 6. Nagai, G., I998, Dante Shinkyoku, Tōkyō, Kōdansha, vol. II, p. I47. 

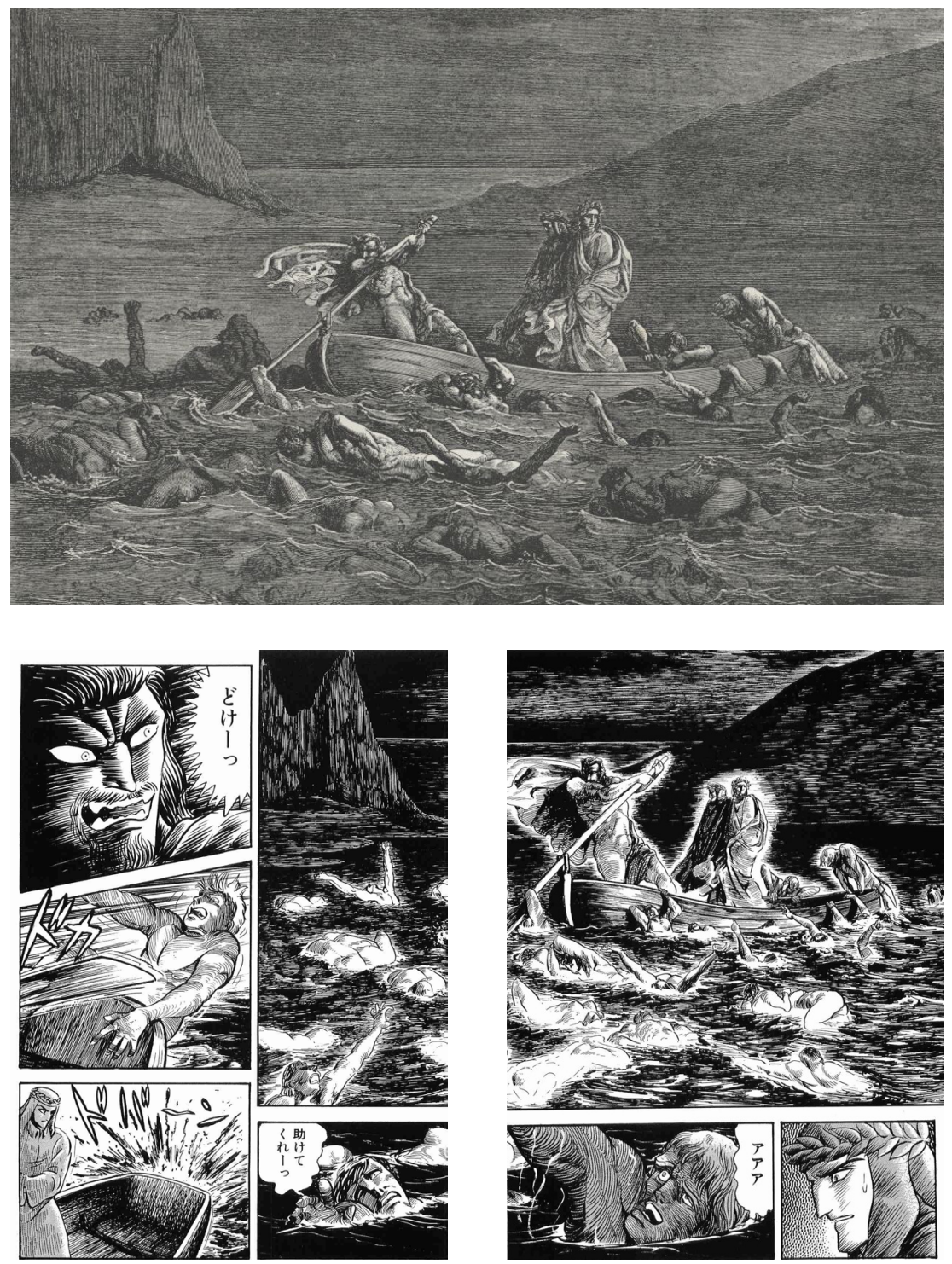

Fig. 7. Gustave Doré, Flegias, If. VIII vv. 29-30; Dante Shinkyoku, vol. I, pp. I86-I9I. 

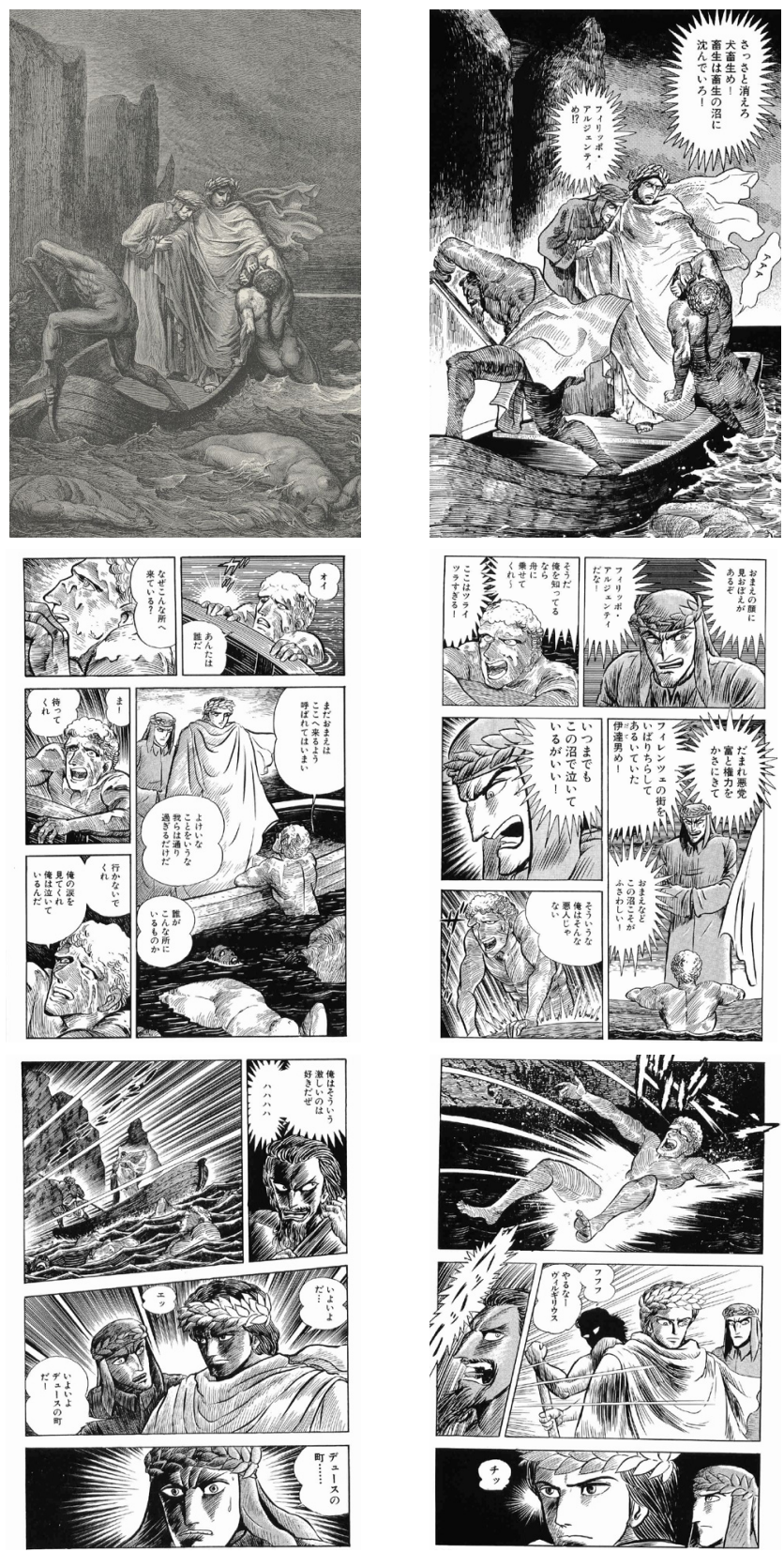

Fig. 8. Gustave Doré, Filippo Argenti, If. VIII vv. 4I-42: Dante Shinkyoku, vol. I, pp. I92-I96. 

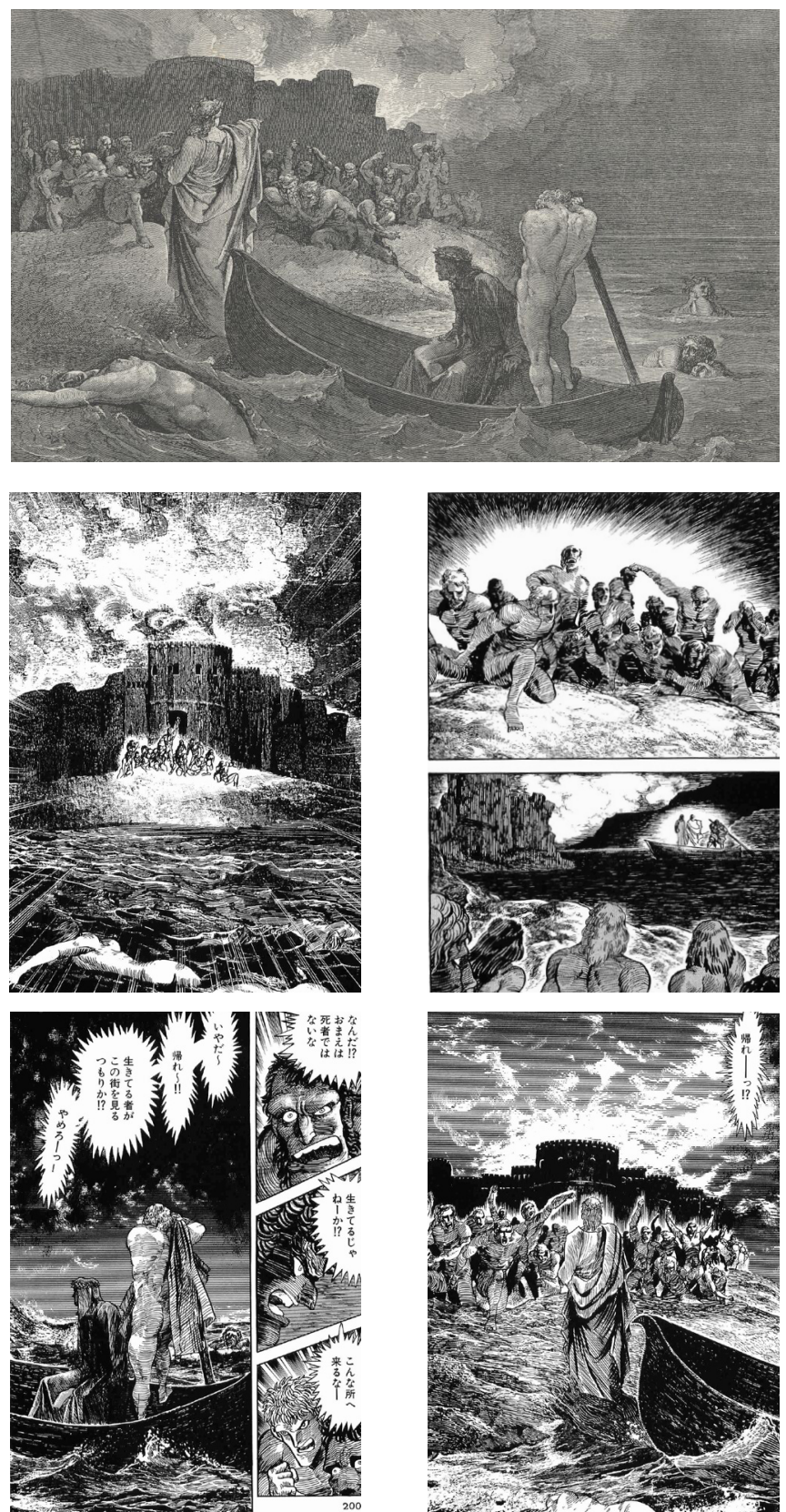

Fig. 9. Gustave Doré, La città di Dite, If. VIII vv. Io9-IIo; Dante Shinkyoku, vol. I, pp. I97, I99-20I. 

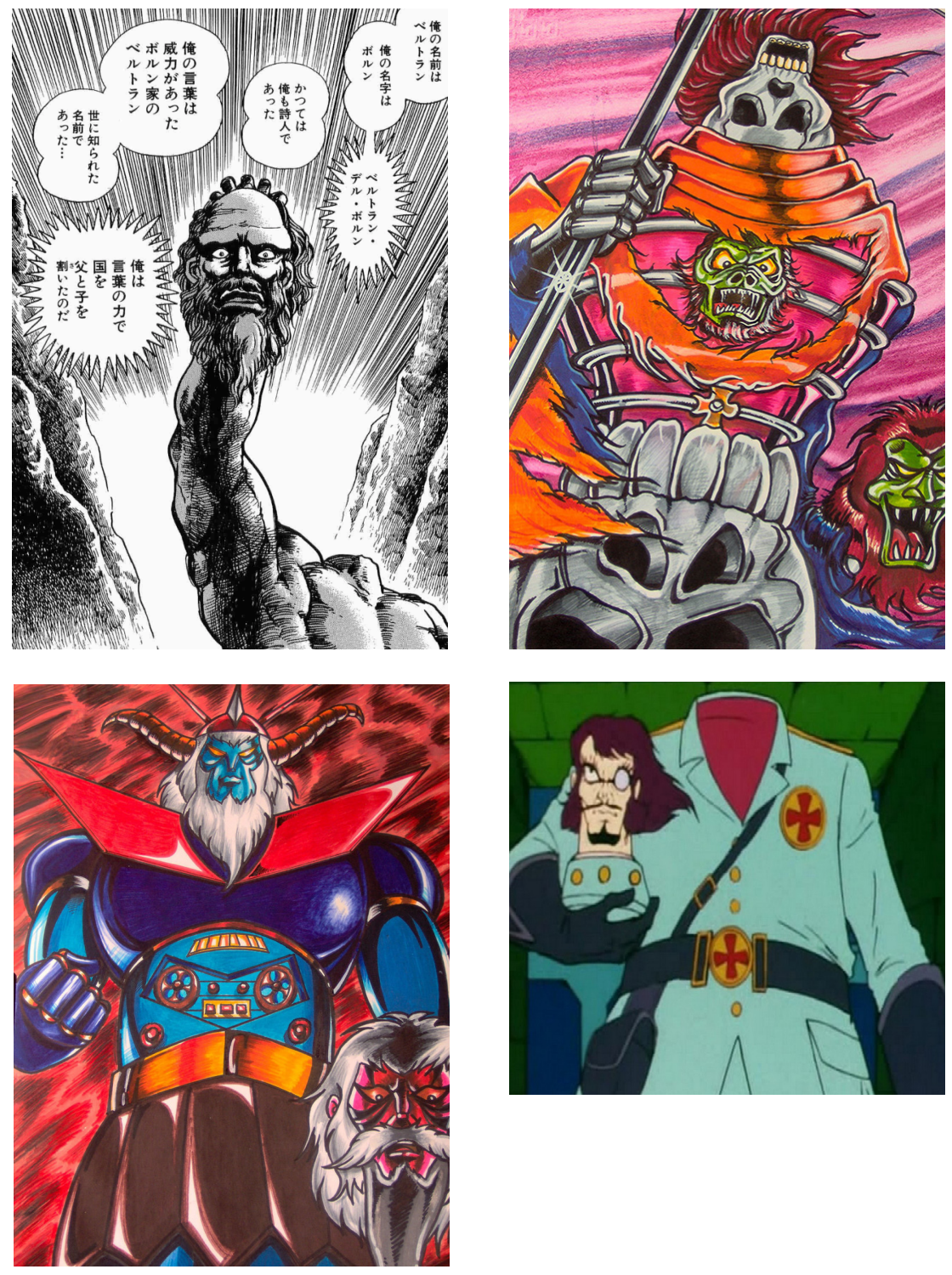

Fig. Io. Dante Shinkyoku, "Bertran de Born", vol. II, p. 46; "Generale Ardias" e "Ministro Argos" da Grande Mazinga (fan art realizzata da Polymar69 su Deviantart, CC3.o); "Conte Blocken" da Mazinga $Z$ (fotogramma). 

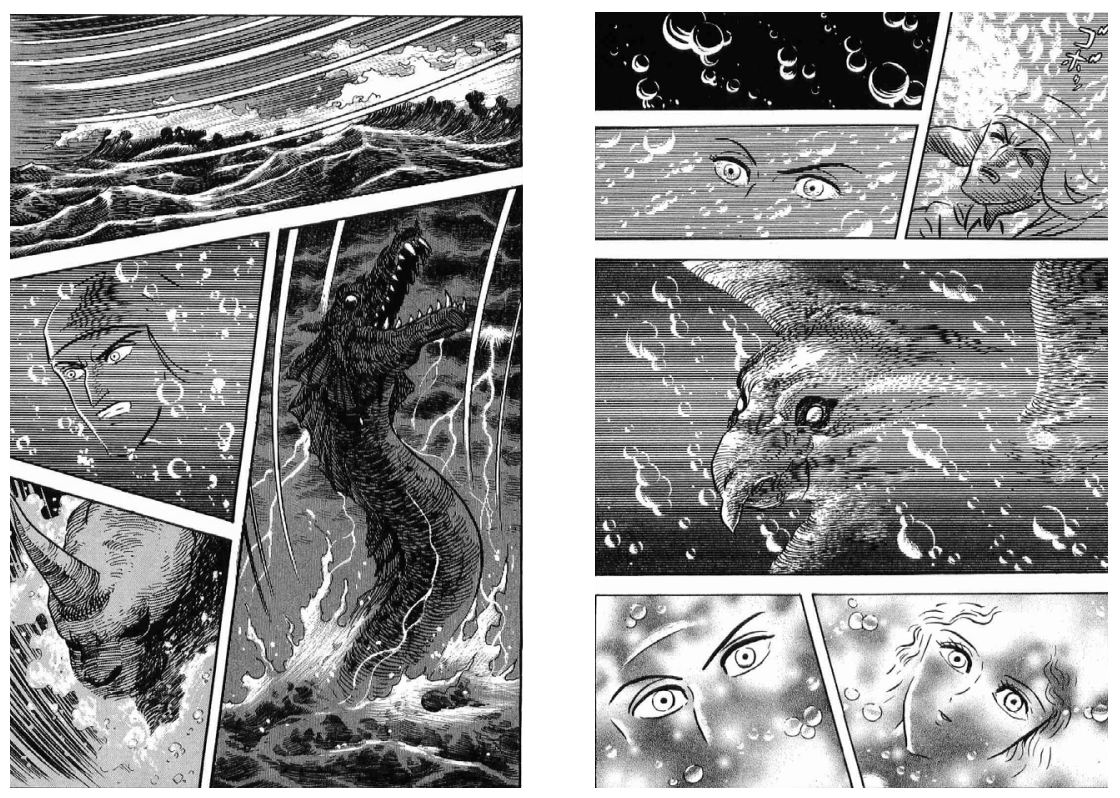

Fig. II. Dante Shinkyoku, vol. II, pp. 348-349. 

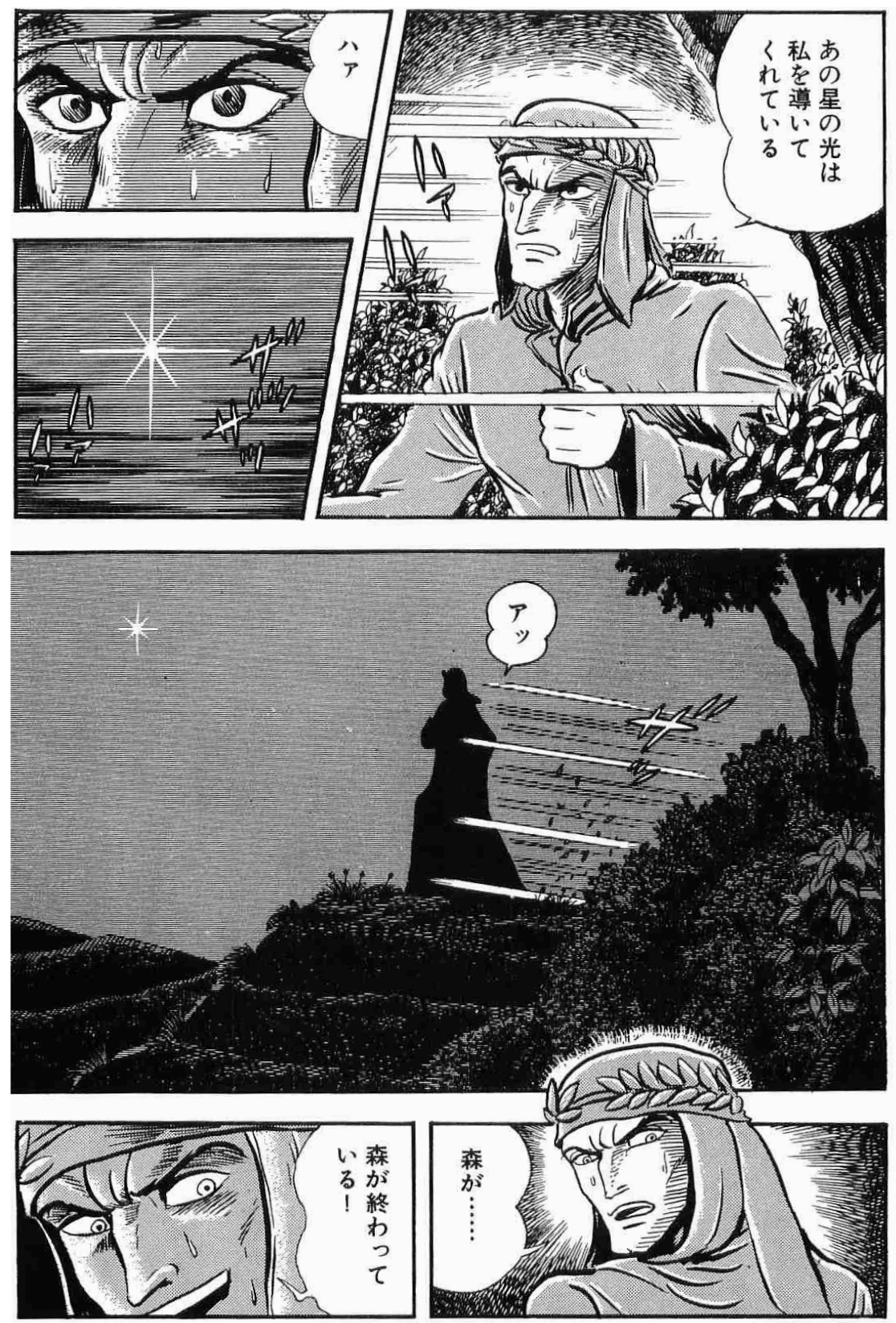

Fig. I2. Dante Shinkyoku, vol. I, p. I3. 
